\title{
ON DECOMPOSITION NUMBERS WITH JANTZEN FILTRATION OF CYCLOTOMIC $q$-SCHUR ALGEBRAS
}

\author{
KENTARO WADA
}

\begin{abstract}
Let $\mathscr{S}(\Lambda)$ be the cyclotomic $q$-Schur algebra associated to the Ariki-Koike algebra $\mathscr{H}_{n, r}$, introduced by Dipper, James and Mathas. In this paper, we consider $v$-decomposition numbers of $\mathscr{S}(\Lambda)$, namely decomposition numbers with respect to the Jantzen filtrations of Weyl modules. We prove, as a $v$-analogue of the result obtained by Shoji and Wada, a product formula for $v$-decomposition numbers of $\mathscr{S}(\Lambda)$, which asserts that certain $v$-decomposition numbers are expressed as a product of $v$-decomposition numbers for various cyclotomic $q$-Schur algebras associated to Ariki-koike algebras $\mathscr{H}_{n_{i}, r_{i}}$ of smaller rank. Moreover, we prove a similar formula for $v$-decomposition numbers of $\mathscr{H}_{n, r}$ by using a Schur functor.
\end{abstract}

\section{INTRODUCTION}

Let $\mathscr{H}=\mathscr{H}_{n, r}$ be the Ariki-Koike algebra over an integral domain $R$ associated to the complex reflection group $\mathfrak{S}_{n} \ltimes(\mathbb{Z} / r \mathbb{Z})^{n}$. Dipper, James and Mathas DJM introduced the cyclotomic $q$-Schur algebra $\mathscr{S}(\Lambda)$ associated to the Ariki-Koike algebra $\mathscr{H}$, and they showed that $\mathscr{H}$ and $\mathscr{S}(\Lambda)$ are cellular algebras in the sense of Graham and Lehrer GL, by constructing the cellular basis respectively. It is a fundamental problem for the representation theory to determine the decomposition numbers of $\mathscr{H}$ and $\mathscr{S}(\Lambda)$. It is well known that the decomposition matrix of $\mathscr{H}$ coincides with the submatrix of that of $\mathscr{S}(\Lambda)$ by the Schur functor.

In the case where $\mathscr{H}$ is the Iwahori-Hecke algebra $\mathscr{H}_{n}$ of type A, Lascoux, Leclerc and Thibon [LLT conjectured that the decomposition numbers of $\mathscr{H}_{n}$ can be described by using the canonical basis of a certain irreducible $U_{v}\left(\widehat{\mathfrak{s l}}_{e}\right)$-module, and gave the algorithm to compute this canonical basis. The cojecture has been solved by Ariki [A1], by extending to the case of Ariki-Koike algebras.

In the case of the $q$-Schur algebra associated to $\mathscr{H}_{n}$, Leclerc and Thibon [LT] conjectured that the decomposition matrix coincides with the transition matrix between the canonical basis and the standard basis of the Fock space of level 1 equipped with the $U_{v}\left(\widehat{\mathfrak{s l}}_{e}\right)$-module structure, and gave the algorithm to compute the transition matrix. This conjecture has been solved by Varagnolo and Vasserot in $\mathrm{VV}$.

More generally, in the case of the cyclotomic $q$-Schur algebra $\mathscr{S}$, Yvonne [Y] has conjectured that the decomposition matrix coincides with the transition matrix between the canonical basis and the standard basis of the higher-level Fock space. This canonical basis was constructed by Uglov [U] and the algorithm to compute the

Received by the editors November 6, 2007.

2010 Mathematics Subject Classification. Primary 20-XX, 16-XX.

(C)2010 American Mathematical Society Reverts to public domain 28 years from publication 
transition matrix was also given there. Yvonne's conjecture is still open. We remark that Ariki's theorem, Varagnolo and Vasserot's theorem, and Yvonne's conjecture are concerned with the situation where $R$ is a complex number field and parameters are roots of unity.

In order to study the decomposition numbers of $\mathscr{S}$, we constructed in [SW] some subalgebras $\mathscr{S}^{\mathbf{p}}$ of $\mathscr{S}(\Lambda)$ and their quotients $\overline{\mathscr{S}}^{\mathbf{p}}$, and showed that $\mathscr{S}^{\mathbf{p}}$ is a standardly based algebra in the sense of Du and Rui [DR], and that $\overline{\mathscr{S}}^{\mathbf{p}}$ is a cellular algebra. Hence, one can consider the decomposition numbers of $\mathscr{S}^{\mathbf{p}}$ and $\overline{\mathscr{S}}^{\mathbf{p}}$ also. We denote the decomposition numbers of $\mathscr{S}, \mathscr{S}^{\mathbf{p}}$ and $\overline{\mathscr{S}}^{\mathbf{p}}$ by $d_{\lambda \mu}, d_{\lambda \mu}^{(\lambda, 0)}$ and $\bar{d}_{\lambda \mu}$ respectively, where $d_{\lambda \mu}$ is a decomposition number of the irreducible module $L^{\mu}$ in the Weyl module $W^{\lambda}$ of $\mathscr{S}$ for $r$-partitions $\lambda, \mu$, and $d_{\lambda \mu}^{(\lambda, 0)}, \bar{d}_{\lambda \mu}$ are defined similarly for $\mathscr{S}^{\mathbf{p}}$ and $\overline{\mathscr{S}}^{\mathbf{p}}$ (see Section 1 for details). It is proved in [SW, Theorem 3.13] that

$$
\bar{d}_{\lambda \mu}=d_{\lambda \mu}^{(\lambda, 0)}=d_{\lambda \mu}
$$

whenever $\lambda, \mu$ satisfy a certain condition $\alpha_{\mathbf{p}}(\lambda)=\alpha_{\mathbf{p}}(\mu)$. Moreover, for such $\lambda, \mu$, the product formula for $\bar{d}_{\lambda \mu}$,

$$
\bar{d}_{\lambda \mu}=\prod_{k=1}^{g} d_{\lambda^{[k]} \mu^{[k]}}
$$

was proved in [SW, Theorem 4.17], where $d_{\lambda^{[k]} \mu^{[k]}}$ for $k=1, \cdots, g$ is the decomposition number of the cyclotomic $q$-Schur algebra associated to a certain Ariki-Koike algebra $\mathscr{H}_{n_{k}, r_{k}}$.

Related to the above conjectures on Fock spaces, Leclerc and Thibon and Yvonne give a more precise conjecture concerning the $v$-decomposition numbers defined by using Jantzen filtrations of Weyl modules. (For definition of $v$-decomposition numbers, see §2.) We remark that decomposition numbers coincide with $v$-decomposition numbers at $v=1$. Thus we regard $v$-decomposition numbers as a $v$-analogue of decomposition numbers. The conjecture for $v$-decomposition numbers is still open even in the case of the $q$-Schur algebra of type A.

In this paper, we show that similar formulas as (1) and (2) also hold for $v$ decomposition numbers. We denote the $v$-decomposition numbers of $\mathscr{S}(\Lambda), \mathscr{S} \mathbf{p}(\Lambda)$ and $\overline{\mathscr{S}}^{\mathbf{p}}(\Lambda)$ by $d_{\lambda \mu}(v), d_{\lambda \mu}^{(\lambda, 0)}(v)$ and $\bar{d}_{\lambda \mu}(v)$ respectively. Then for $r$-partitions $\lambda, \mu$ such that $\alpha_{\mathbf{p}}(\lambda)=\alpha_{\mathbf{p}}(\mu)$, we have (Theorem 2.8)

$$
\bar{d}_{\lambda \mu}(v)=d_{\lambda \mu}^{(\lambda, 0)}(v)=d_{\lambda \mu}(v)
$$

and (Theorem 2.14)

$$
d_{\lambda \mu}(v)=\bar{d}_{\lambda \mu}(v)=\prod_{k=1}^{g} d_{\lambda^{[k]} \mu^{[k]}}(v),
$$

where $d_{\lambda^{[k]} \mu^{[k]}}(v)$ is the $v$-decomposition number of the cyclotomic $q$-Schur algebra appeared in (2).

We note that our result is a $v$-analogue of (1) and (2), and it reduces to them by taking $v \mapsto 1$. Moreover, for a certain $v$-decomposition number $d_{\lambda \mu}^{\mathscr{H}}(v)$ of the Ariki-Koike algebra, we also have the following product formula (Theorem 3.5).

$$
d_{\lambda \mu}^{\mathscr{H}}(v)=\prod_{k=1}^{g} d_{\lambda^{[k]} \mu^{[k]}}^{\mathscr{H}}(v),
$$


where $d_{\lambda^{[k]} \mu^{[k]}}^{\mathscr{H}}(v)$ is the $v$-decomposition number of a certain Ariki-Koike algebra $\mathscr{H}_{n_{k}, r_{k}}$.

We remark that our results hold for any parameters and any modular system, even for the case where the base field has non-zero characteristic, though Yvonne's conjecture is formulated under certain restrictions for parameters and modular systems.

\section{A REVIEW OF KNOWN RESULTS}

1.1. Througout this paper, we follow the notation in $[\mathrm{SW}$. Here we review some of them. We fix positive integers $r, n$ and an $r$-tuple $\mathbf{m}=\left(m_{1}, \cdots, m_{r}\right) \in \mathbb{Z}_{>0}^{r}$. A composition $\lambda=\left(\lambda_{1}, \lambda_{2}, \cdots\right)$ is a finite sequence of non-negative integers, and $|\lambda|=\sum_{i} \lambda_{i}$ is called the size of $\lambda$. If $\lambda_{l} \neq 0$ and $\lambda_{k}=0$ for any $k>l$, then $l$ is called the length of $\lambda$. If the composition $\lambda$ is a weakly decreasing sequence, $\lambda$ is called a partition. An $r$-tuple $\mu=\left(\mu^{(1)}, \cdots, \mu^{(r)}\right)$ of compositions is called the $r$-composition, and size $|\mu|$ of $\mu$ is defined by $\sum_{i=1}^{r}\left|\mu^{(i)}\right|$. In particular, if all $\mu^{(i)}$ are partitions, $\mu$ is called an $r$-partition. We denote by $\Lambda=\widetilde{\mathcal{P}}_{n, r}(\mathbf{m})$ the set of $r$-compositions $\mu=\left(\mu^{(1)}, \cdots, \mu^{(r)}\right)$ such that $|\mu|=n$ and that the length of $\mu^{(k)}$ is smaller than $m_{k}$ for $k=1, \cdots, r$. We define $\Lambda^{+}=\mathcal{P}_{n, r}(\mathbf{m})$ as the subset of $\Lambda$ consisting of $r$-partitions.

We define the partial order, the so-called "dominance order", on $\Lambda$ by $\mu \unrhd \nu$ if and only if

$$
\sum_{i=1}^{l}\left|\mu^{(i)}\right|+\sum_{j=1}^{k} \mu_{j}^{(l)} \geq \sum_{i=1}^{l}\left|\nu^{(i)}\right|+\sum_{j=1}^{k} \nu_{j}^{(l)}
$$

for any $1 \leq l \leq r, 1 \leq k \leq m_{l}$. If $\mu \unrhd \nu$ and $\mu \neq \nu$, we write it as $\mu \triangleright \nu$.

For $\lambda \in \Lambda^{+}$, we denote by $\operatorname{Std}(\lambda)$ the set of standard tableau of shape $\lambda$. For $\lambda \in \Lambda^{+}$and $\mu \in \Lambda$, we denote by $\mathcal{T}_{0}(\lambda, \mu)$ the set of semistandard $\lambda$-tableau of type $\mu$. Moreover, we set $\mathcal{T}_{0}(\lambda)=\bigcup_{\mu \in \Lambda} \mathcal{T}_{0}(\lambda, \mu)$. For definitions of standard tableau and semistandard tableau, see [SW] or [DJM].

1.2. Let $\mathscr{H}=\mathscr{H}_{n, r}$ be the Ariki-Koike algebra over an integral domain $R$ with parameters $q, Q_{1}, \cdots, Q_{r}$ with defining relations in [SW, $\left.\S 1.1\right]$. It is known by [DJM] that $\mathscr{H}$ has a structure of the cellular algebra with a cellular basis $\left\{m_{\mathfrak{s t}} \mid \mathfrak{s}, \mathfrak{t} \in\right.$ $\operatorname{Std}(\lambda)$ for some $\left.\lambda \in \Lambda^{+}\right\}$. Then the general theory of a cellular algebra by GL implies the following results. There exists an anti-automorphism $h \mapsto h^{*}$ of $\mathscr{H}$ such that $m_{\mathfrak{s t}}^{*}=m_{\mathfrak{t} \mathfrak{s}}$. For $\lambda \in \Lambda^{+}$, let $\mathscr{H}^{\vee \lambda}$ be the $R$-submodule of $\mathscr{H}$ spanned by $m_{\mathfrak{s t}}$, where $\mathfrak{s}, \mathfrak{t} \in \operatorname{Std}(\mu)$ for some $\mu \in \Lambda^{+}$such that $\mu \triangleright \lambda$. Then $\mathscr{H}^{\vee \lambda}$ is an ideal of $\mathscr{H}$. One can construct the standard (right) $\mathscr{H}$-module $S^{\lambda}$, called a Specht module, with the $R$-free basis $\left\{m_{\mathfrak{t}} \mid \mathfrak{t} \in \operatorname{Std}(\lambda)\right\}$. We define the bilinear form $\langle,\rangle_{\mathscr{H}}$ on $S^{\lambda}$ by

$$
\left\langle m_{\mathfrak{s}}, m_{\mathfrak{t}}\right\rangle_{\mathscr{H}} m_{\mathfrak{u v}} \equiv m_{\mathfrak{u s}} m_{\mathfrak{t v}} \quad \bmod \mathscr{H}^{\vee \lambda} \quad(\mathfrak{s}, \mathfrak{t} \in \operatorname{Std}(\lambda))
$$

where $\mathfrak{u}, \mathfrak{v} \in \operatorname{Std}(\lambda)$, and the scalar $\left\langle m_{\mathfrak{s}}, m_{\mathfrak{t}}\right\rangle_{\mathscr{H}}$ does not depend on the choice of $\mathfrak{u}, \mathfrak{v} \in \operatorname{Std}(\lambda)$. The bilinear form $\langle,\rangle_{\mathscr{H}}$ is associative, namely we have

$$
\langle x h, y\rangle_{\mathscr{H}}=\left\langle x, y h^{*}\right\rangle_{\mathscr{H}} \quad \text { for } x, y \in S^{\lambda}, h \in \mathscr{H} \text {. }
$$

Let $\operatorname{rad} S^{\lambda}=\left\{x \in S^{\lambda} \mid\langle x, y\rangle_{\mathscr{H}}=0\right.$ for any $\left.y \in S^{\lambda}\right\}$. Then $\operatorname{rad} S^{\lambda}$ is the $\mathscr{H}$ submodule of $S^{\lambda}$ by the associativity of the bilinear form. Put $D^{\lambda}=S^{\lambda} / \operatorname{rad} S^{\lambda}$. Assume that $R$ is a field. Then $D^{\lambda}$ is an absolutely irreducible module or zero, and 
the set $\left\{D^{\lambda} \mid \lambda \in \Lambda^{+}\right.$such that $\left.D^{\lambda} \neq 0\right\}$ gives a complete set of non-isomorphic irreducible $\mathscr{H}$-modules.

1.3. Let $\mathscr{S}=\mathscr{S}(\Lambda)$ be the cyclotomic $q$-Schur algebra introduced by [DJM], associated to the Ariki-Koike algebra $\mathscr{H}$ with respect to the set $\Lambda$. It is known by [DJM] that $\mathscr{S}$ is a cellular algebra with a cellular basis $\left\{\varphi_{S T} \mid S, T \in \mathcal{T}_{0}(\lambda)\right.$ for some $\lambda \in$ $\left.\Lambda^{+}\right\}$. Again by the general theory of a cellular algebra, the following results hold. There exists the anti-automorphism $x \mapsto x^{*}$ of $\mathscr{S}$ such that $\varphi_{S T}^{*}=\varphi_{T S}$. For $\lambda \in \Lambda^{+}$, let $\mathscr{S}^{\vee \lambda}$ be the $R$-submodule spanned by $\varphi_{S T}$, where $S, T \in \mathcal{T}_{0}(\mu)$ for some $\mu \in \Lambda^{+}$such that $\mu \triangleright \lambda$. Then $\mathscr{S}^{\vee \lambda}$ is an ideal of $\mathscr{S}$. One can construct the standard (right) $\mathscr{S}$-module $W^{\lambda}\left(\lambda \in \Lambda^{+}\right)$, called a Weyl module, with the $R$-free basis $\left\{\varphi_{T} \mid T \in \mathcal{T}_{0}(\lambda)\right\}$. We define a bilinear form $\langle$,$\rangle on W^{\lambda}$ by

$$
\left\langle\varphi_{S}, \varphi_{T}\right\rangle \varphi_{U V} \equiv \varphi_{U S} \varphi_{T V} \quad \bmod \mathscr{S}^{\vee \lambda} \quad\left(S, T \in \mathcal{T}_{0}(\lambda)\right)
$$

where $U, V \in \mathcal{T}_{0}(\lambda)$, and the scalar $\left\langle\varphi_{S}, \varphi_{T}\right\rangle$ does not depend on a choice of $U, V \in$ $\mathcal{T}_{0}(\lambda)$. The bilinear form $\langle$,$\rangle is associative, namely we have$

$$
\langle x \varphi, y\rangle=\left\langle x, y \varphi^{*}\right\rangle \quad \text { for } x, y \in W^{\lambda}, \varphi \in \mathscr{S} \text {. }
$$

Let $\operatorname{rad} W^{\lambda}=\left\{x \in W^{\lambda} \mid\langle x, y\rangle=0\right.$ for any $\left.y \in W^{\lambda}\right\}$, Then $\operatorname{rad} W^{\lambda}$ is the $\mathscr{S}$ submodule of $W^{\lambda}$. Put $L^{\lambda}=W^{\lambda} / \operatorname{rad} W^{\lambda}$. Then it is known by DJM] that $L^{\lambda} \neq 0$ for any $\lambda \in \Lambda^{+}$. Assume that $R$ is a field. Then $L^{\lambda}$ is an absolutely irreducible module, and the set $\left\{L^{\lambda} \mid \lambda \in \Lambda^{+}\right\}$gives a complete set of non-isomorphic irreducible $\mathscr{S}$-modules.

1.4. We recall some definitions and results in $[\mathrm{SW}]$. We fix a positive integer $g \leq r$ and $\mathbf{p}=\left(r_{1}, \cdots, r_{g}\right) \in \mathbb{Z}_{>0}^{g}$ such that $r_{1}+\cdots+r_{g}=r$, and set $p_{1}=0, p_{i}=\sum_{j=1}^{i-1} r_{j}$ for $i=2, \cdots, g$. For $\mu=\left(\mu^{(1)}, \cdots, \mu^{(r)}\right) \in \Lambda$, we define $\alpha_{\mathbf{p}}(\mu)=\left(n_{1}, \cdots, n_{g}\right)$ and $\mathbf{a}_{\mathbf{p}}(\mu)=\left(a_{1}, \cdots, a_{g}\right)$, where $n_{k}=\sum_{i=1}^{r_{k}}\left|\mu^{\left(p_{k}+i\right)}\right|$ and $a_{k}=\sum_{i=1}^{k-1} n_{i}$ for $k=$ $1, \cdots, g$ with $a_{1}=0$. We define a partial order on $\mathbb{Z}_{>0}^{g}$ by $\mathbf{a}=\left(a_{1}, \cdots, a_{g}\right) \geq \mathbf{b}=$ $\left(b_{1}, \cdots, b_{g}\right)$ if $a_{i} \geq b_{i}$ for any $i=1, \cdots, g$ and we write $\mathbf{a}>\mathbf{b}$ if $\mathbf{a} \geq \mathbf{b}$ and $\mathbf{a} \neq \mathbf{b}$. Later we consider the partial order on $\left\{\mathbf{a}_{\mathbf{p}}(\mu) \mid \mu \in \Lambda\right\}$ by this order.

For $\lambda \in \Lambda^{+}$and $\mu \in \Lambda$, we set $\mathcal{T}_{0}^{\mathbf{p}}(\lambda, \mu)=\mathcal{T}_{0}(\lambda, \mu)$ if $\alpha_{\mathbf{p}}(\lambda)=\alpha_{\mathbf{p}}(\mu)$, and it is empty otherwise. Moreover, we set $\mathcal{T}_{0}^{\mathbf{p}}(\lambda)=\bigcup_{\mu \in \Lambda} \mathcal{T}_{0}^{\mathbf{p}}(\lambda, \mu)$. We set

$$
\begin{aligned}
\Sigma^{\mathbf{p}}=\left(\Lambda^{+} \times\{0,1\}\right) \backslash\{(\lambda, 1) & \in \Lambda^{+} \times\{0,1\} \mid \mathcal{T}_{0}(\lambda, \mu)=\phi \\
& \text { for any } \left.\mu \in \Lambda \text { such that } \mathbf{a}_{\mathbf{p}}(\lambda)>\mathbf{a}_{\mathbf{p}}(\mu)\right\},
\end{aligned}
$$

and define a partial order $\geq$ on $\Sigma \mathbf{p}$ by $\left(\lambda_{1}, \varepsilon_{1}\right)>\left(\lambda_{2}, \varepsilon_{2}\right)$ if $\lambda_{1} \triangleright \lambda_{2}$ or if $\lambda_{1}=\lambda_{2}$ and $\varepsilon_{1}>\varepsilon_{2}$. For $\eta=(\lambda, \varepsilon) \in \Sigma^{\mathbf{p}}$, we set

$$
\begin{gathered}
I(\eta)= \begin{cases}\mathcal{T}_{0}^{\mathbf{p}}(\lambda) & \text { if } \varepsilon=0, \\
\bigcup_{\substack{\mu \in \Lambda \\
\mathbf{a}_{\mathbf{p}}(\lambda)>\mathbf{a}_{\mathbf{p}}(\mu)}} \mathcal{T}_{0}(\lambda, \mu) & \text { if } \varepsilon=1,\end{cases} \\
J(\eta)= \begin{cases}\mathcal{T}_{0}^{\mathbf{p}}(\lambda) & \text { if } \varepsilon=0, \\
\mathcal{T}_{0}(\lambda) & \text { if } \varepsilon=1,\end{cases} \\
\mathcal{C}^{\mathbf{p}}(\eta)=\left\{\varphi_{S T} \mid(S, T) \in I(\eta) \times J(\eta)\right\}
\end{gathered}
$$


and

$$
\mathcal{C}^{\mathbf{p}}=\bigcup_{\eta \in \Sigma^{\mathbf{p}}} \mathcal{C}^{\mathbf{p}}(\eta)
$$

Let $\mathscr{S}^{\mathbf{p}}=\mathscr{S}^{\mathbf{p}}(\Lambda)$ be the $R$-submodule of $\mathscr{S}(\Lambda)$ spanned by $\mathcal{C}^{\mathbf{p}}$. We also define $\left(\mathscr{S}^{\mathbf{p}}\right)^{\vee \eta}$ as the $R$-submodule of $\mathscr{S}^{\mathbf{p}}$ spanned by

$$
\left\{\varphi_{U V} \mid(U, V) \in I\left(\eta^{\prime}\right) \times J\left(\eta^{\prime}\right) \text { for some } \eta^{\prime} \in \Sigma^{\mathbf{p}} \text { such that } \eta^{\prime}>\eta\right\} .
$$

It is known by [SW, Theorem 2.6] that $\mathscr{S}^{\mathbf{p}}$ is a standardly based algebra with the standard basis $\mathcal{C}^{\mathbf{p}}$ in the sense of $[\mathrm{DR}$.

By the general theory of standardly based algebras due to [DR], we have the following results. For $\eta \in \Sigma^{\mathbf{p}}$, one can consider the standard left $\mathscr{S}^{\mathbf{p}}$-modules $\diamond Z^{\eta}$ with the basis $\left\{\varphi_{T}^{\eta} \mid T \in I(\eta)\right\}$ and the standard right $\mathscr{S}^{\mathbf{p}}$-module $Z^{\eta}$ with the basis $\left\{\varphi_{T}^{\eta} \mid T \in J(\eta)\right\}$. We call them Weyl modules of $\mathscr{S}^{\mathbf{p}}$. We define the bilinear form $\beta_{\eta}: \diamond Z^{\eta} \times Z^{\eta} \rightarrow R$ by

$$
\beta_{\eta}\left(\varphi_{S}^{\eta}, \varphi_{T}^{\eta}\right) \varphi_{U V} \equiv \varphi_{U T} \varphi_{S V} \quad \bmod \left(\mathscr{S}^{\mathbf{p}}\right)^{\vee \eta} \quad(S \in I(\eta), T \in J(\eta)),
$$

where $\beta_{\eta}$ is determined independent of the choice of $U \in I(\eta)$ and $V \in J(\eta)$. The bilinear form $\beta_{\eta}$ is associative, namely we have

$$
\beta_{\eta}(\varphi x, y)=\beta_{\eta}(x, y \varphi) \quad \text { for } x \in \nabla^{\eta}, y \in Z^{\eta}, \varphi \in \mathscr{S}^{\mathbf{p}} \text {. }
$$

Let $\operatorname{rad} Z^{\eta}=\left\{x \in Z^{\eta} \mid \beta_{\eta}(y, x)=0\right.$ for any $\left.y \in \diamond Z^{\eta}\right\}$. Then $\operatorname{rad} Z^{\eta}$ is a $\mathscr{S}^{\mathbf{p}}$ submodule of $Z^{\eta}$ by associativity of $\beta_{\eta}$. Put $L^{\eta}=Z^{\eta} / \operatorname{rad} Z^{\eta}$. Assume that $R$ is a field. Then $L^{\eta}$ is an absolutely irreducible module or zero, and the set $\left\{L^{\eta} \mid \eta \in \Sigma^{\mathbf{p}}\right.$ such that $\left.\beta_{\eta} \neq 0\right\}$ is a complete set of non-isomorphic irreducible (right) $\mathscr{S}^{\mathbf{p}}$-modules.

Later we shall only consider the Weyl modules $Z^{\eta}$ and irreducible modules $L^{\eta}$ of $\mathscr{S}^{\mathbf{p}}$ for $\eta$ of the form $(\lambda, 0)$. Note that the composition fuctors of $Z^{(\lambda, 0)}$ are isomorphic to $L^{(\mu, 0)}$ for some $\mu \in \Lambda^{+}$by [SW, Proposition 3.3 (i)].

1.5. Let $\widehat{\mathscr{S}^{\mathbf{p}}}$ be the $R$-submodule of $\mathscr{S}^{\mathbf{p}}$ spanned by

$$
\mathcal{C}^{\mathbf{p}} \backslash\left\{\varphi_{S T} \mid S, T \in \mathcal{T}_{0}^{\mathbf{p}}(\lambda) \text { for some } \lambda \in \Lambda^{+}\right\} .
$$

It is known by $[\mathrm{SW}]$ that $\widehat{\mathscr{S}^{\mathbf{p}}}$ is a two-sided ideal of $\mathscr{S}^{\mathbf{p}}$. Thus, we can define the quotient algebra

$$
\overline{\mathscr{S}}^{\mathbf{p}}=\mathscr{S}^{\mathbf{p}} / \widehat{\mathscr{S}^{\mathbf{p}}} \text {. }
$$

We denote by $\bar{\varphi}$ the image of $\varphi \in \mathscr{S}^{\mathbf{p}}$ under the natural surjection $\pi: \mathscr{S}^{\mathbf{p}} \rightarrow \overline{\mathscr{S}}^{\mathbf{p}}$, and set

$$
\overline{\mathcal{C}}^{\mathbf{p}}=\left\{\bar{\varphi}_{S T} \mid S, T \in \mathcal{T}_{0}^{\mathbf{p}}(\lambda) \text { for some } \lambda \in \Lambda^{+}\right\} .
$$

Then $\overline{\mathcal{C}}^{\mathbf{p}}$ is a free $R$-basis of $\overline{\mathscr{S}}^{\mathbf{p}}$. By [SW, Theorem 2.13], $\overline{\mathscr{S}}^{\mathbf{p}}$ turns out to be a cellular algebra with the cellular basis $\overline{\mathcal{C}}^{\mathbf{p}}$. Hence by the general theory of cellular algebra, the following results hold. For $\lambda \in \Lambda^{+}$, we can consider the standard (right) $\overline{\mathscr{S}}^{\mathrm{p}}$-module $\bar{Z}^{\lambda}$ with the free $R$-basis $\left\{\bar{\varphi}_{T} \mid T \in \mathcal{T}_{0}^{\mathrm{p}}(\lambda)\right\}$. We call it a Weyl module of $\overline{\mathscr{S}}^{\mathbf{p}}$. We define the bilinear form $\langle,\rangle_{\mathbf{p}}: \bar{Z}^{\lambda} \times \bar{Z}^{\lambda} \rightarrow R$ by

$$
\left\langle\bar{\varphi}_{S}, \bar{\varphi}_{T}\right\rangle_{\mathbf{p}} \bar{\varphi}_{U V} \equiv \bar{\varphi}_{U S} \bar{\varphi}_{T V} \quad \bmod \left(\overline{\mathscr{S}}^{\mathbf{p}}\right)^{\vee \lambda} \quad\left(S, T \in \mathcal{T}_{0}^{\mathbf{p}}(\lambda)\right)
$$

where $\langle,\rangle_{\mathbf{p}}$ is determined independently of the choice $U, V \in \mathcal{T}_{0}^{\mathbf{p}}(\lambda)$, and $\left(\overline{\mathscr{S}}^{\mathbf{p}}\right)^{\vee \lambda}$ is the $R$-submodule of $\overline{\mathscr{S}}^{\mathbf{p}}$ spanned by

$$
\left\{\bar{\varphi}_{S T} \mid S, T \in \mathcal{T}_{0}^{\mathbf{P}}\left(\lambda^{\prime}\right) \text { for some } \lambda^{\prime} \in \Lambda^{+} \text {such that } \lambda^{\prime} \triangleright \lambda\right\} \text {. }
$$


The bilinear form $\langle,\rangle_{\mathbf{p}}$ is associative, namely we have

$$
\langle\bar{x} \bar{\varphi}, \bar{y}\rangle_{\mathbf{p}}=\left\langle\bar{x}, \bar{y} \bar{\varphi}^{*}\right\rangle_{\mathbf{p}} \quad \text { for any } \bar{x}, \bar{y} \in \bar{Z}^{\lambda}, \bar{\varphi} \in \overline{\mathscr{S}}^{\mathbf{p}} .
$$

Let $\operatorname{rad} \bar{Z}^{\lambda}=\left\{\bar{x} \in \bar{Z}^{\lambda} \mid\langle\bar{x}, \bar{y}\rangle_{\mathbf{p}}=0\right.$ for any $\left.\bar{y} \in \bar{Z}^{\lambda}\right\}$, then $\operatorname{rad} \bar{Z}^{\lambda}$ is an $\overline{\mathscr{S}}^{\mathbf{p}}$ submodule of $\bar{Z}^{\lambda}$. Put $\bar{L}^{\lambda}=\bar{Z}^{\lambda} / \operatorname{rad} \bar{Z}^{\lambda}$. Assume that $R$ is a field. Then $\bar{L}^{\lambda}$ is an absolutely irreducible module, and the set $\left\{\bar{L}^{\lambda} \mid \lambda \in \Lambda^{+}\right\}$is a complete set of non-isomorphic irreducible (right) $\overline{\mathscr{S}}^{\mathrm{p}}$-modules.

1.6. Assuming that $R$ is a field, we set, for $\lambda, \mu \in \Lambda^{+}$,

$$
\begin{aligned}
& d_{\lambda \mu}=\left[W^{\lambda}: L^{\mu}\right], \\
& d_{\lambda \mu}^{(\lambda, 0)}=\left[Z^{(\lambda, 0)}: L^{(\mu, 0)}\right], \\
& \bar{d}_{\lambda \mu}=\left[\bar{Z}^{\lambda}: \bar{L}^{\mu}\right],
\end{aligned}
$$

where $\left[W^{\lambda}: L^{\mu}\right]$ is the decomposition number of $L^{\mu}$ in $W^{\lambda}$, and similarly for $\mathscr{S}^{\mathbf{p}}$ and $\overline{\mathscr{S}}^{\mathbf{P}}$. The following theorem was proved in $\underline{\mathrm{SW}}$.

Theorem 1.7 ([SW] Theorem 3.13]). Assume that $R$ is a field. For $\lambda, \mu \in \Lambda^{+}$ such that $\alpha_{\mathbf{p}}(\lambda)=\alpha_{\mathbf{p}}(\mu)$, we have

$$
\bar{d}_{\lambda \mu}=d_{\lambda \mu}^{(\lambda, 0)}=d_{\lambda \mu},
$$

1.8. For $\mu=\left(\mu^{(1)}, \cdots, \mu^{(r)}\right) \in \Lambda$, we write it in the form $\mu=\left(\mu^{[1]}, \cdots, \mu^{[g]}\right)$, where $\mu^{[i]}=\left(\mu^{\left(p_{i}+1\right)}, \cdots, \mu^{\left(p_{i}+r_{i}\right)}\right)$. According to the expression of $\mu$ as above, $T=\left(T^{(1)}, \cdots, T^{(r)}\right) \in \mathcal{T}_{0}(\lambda)$ can be expressed as $T=\left(T^{[1]}, \cdots, T^{[g]}\right)$ with $T^{[i]}=$ $\left(T^{\left(p_{i}+1\right)}, \cdots, T^{\left(p_{i}+r_{i}\right)}\right)$. By [SW Lemma 4.3 (iii)], we have a bijection $\mathcal{T}_{0}^{\mathbf{P}}(\lambda, \mu) \simeq$ $\mathcal{T}_{0}\left(\lambda^{[1]}, \mu^{[1]}\right) \times \cdots \times \mathcal{T}_{0}\left(\lambda^{[g]}, \mu^{[g]}\right)$ given by the map $T \mapsto\left(T^{[1]}, \cdots, T^{[g]}\right)$. Thus we have a bijection $\mathcal{T}_{0}^{\mathbf{p}}(\lambda) \simeq \mathcal{T}_{0}\left(\lambda^{[1]}\right) \times \cdots \times \mathcal{T}_{0}\left(\lambda^{[g]}\right)$.

We write $\mathbf{m}=\left(m_{1}, \cdots, m_{r}\right)$ in the form $\mathbf{m}=\left(\mathbf{m}^{[1]}, \cdots, \mathbf{m}^{[g]}\right)$, where $\mathbf{m}^{[k]}=$ $\left(m_{p_{k}+1}, \cdots, m_{p_{k}+r_{k}}\right)$. For each $n_{k} \in \mathbb{Z}_{\geq 0}$, put $\Lambda_{n_{k}}=\widetilde{\mathcal{P}}_{n_{k}, r_{k}}\left(\mathbf{m}^{[k]}\right)$, and $\Lambda_{n_{k}}^{+}=$ $\mathcal{P}_{n_{k}, r_{k}}\left(\mathbf{m}^{[k]}\right)$. $\left(\Lambda_{n_{k}}\right.$ or $\Lambda_{n_{k}}^{+}$is regarded as the empty set if $n_{k}=0$.) Let $\mathscr{S}\left(\Lambda_{n_{k}}\right)$ be the cyclotomic $q$-Schur algebra associated to the Ariki-Koike algebra $\mathscr{H}_{n_{k}, r_{k}}$ with parameters $q, Q_{p_{k}+1}, \cdots, Q_{p_{k}+r_{k}}$. Let $\Delta_{n, g}$ be the set of $\left(n_{1}, \cdots, n_{g}\right) \in \mathbb{Z}_{\geq 0}^{g}$ such that $n_{1}+\cdots+n_{g}=n$. Then we have the following decomposition theorem of $\overline{\mathscr{S}}^{\mathbf{p}}$ by [SW, Theorem 4.15]:

$$
\overline{\mathscr{S}}^{\mathbf{p}}(\Lambda) \cong \bigoplus_{\left(n_{1}, \cdots, n_{g}\right) \in \Delta_{n, g}} \mathscr{S}\left(\Lambda_{n_{1}}\right) \otimes \cdots \otimes \mathscr{S}\left(\Lambda_{n_{g}}\right) \quad \text { as an R-algebra }
$$

under the isomorphism given by

$$
\bar{\varphi}_{S T} \mapsto \varphi_{S^{[1]} T^{[1]}} \otimes \cdots \otimes \varphi_{S^{[g]} T^{[g]}} \quad \text { for } S, T \in \mathcal{T}_{0}^{\mathbf{p}}(\lambda) .
$$

Assuming that $R$ is a field, for $\lambda^{[k]} \in \Lambda_{n_{k}}$, let $W^{\lambda^{[k]}}$ be the Weyl module of $\mathscr{S}\left(\Lambda_{n_{k}}\right)$, and $L^{\lambda^{[k]}}=W^{\lambda^{[k]}} / \operatorname{rad} W^{\lambda^{[k]}}$ the irreducible module. By [SW] Corollary 4.16 ], the following properties hold. Under the isomorphism in (1.8.1), we have, 
for $\lambda, \mu \in \Lambda^{+}$,

$$
\begin{aligned}
& \bar{Z}^{\lambda} \cong W^{\lambda^{[1]}} \otimes \cdots \otimes W^{\lambda^{[g]}}, \\
& \bar{L}^{\mu} \cong L^{\mu^{[1]}} \otimes \cdots \otimes L^{\mu^{[g]}}, \\
& {\left[\bar{Z}^{\lambda}: \bar{L}^{\mu}\right]= \begin{cases}\prod_{k=1}^{g}\left[W^{\lambda^{[k]}}: L^{\mu^{[k]}}\right] & \text { if } \alpha_{\mathbf{p}}(\lambda)=\alpha_{\mathbf{p}}(\mu), \\
0 & \text { otherwise. }\end{cases} }
\end{aligned}
$$

Under the isomorphism in (1.8.3), a bilinear form $\langle,\rangle_{\mathbf{P}}$ on $\bar{Z}^{\lambda}$ decomposes to a product of bilinear forms on $W^{\lambda^{[k]}}$ for $k=1, \cdots, g$, namely we have the following lemma.

Lemma 1.9. For $S, T \in \mathcal{T}_{0}^{\mathbf{p}}(\lambda)$, we have

$$
\left\langle\bar{\varphi}_{S}, \bar{\varphi}_{T}\right\rangle_{\mathbf{p}}=\left\langle\varphi_{S^{[1]}}, \varphi_{T^{[1]}}\right\rangle \cdots\left\langle\varphi_{S^{[g]}}, \varphi_{T^{[g]}}\right\rangle,
$$

where $\left\langle\varphi_{S^{[k]}}, \varphi_{T^{[k]}}\right\rangle$ denotes the bilinear form on $W^{\lambda^{[k]}}$ for $k=1, \cdots, g$.

Proof. Fix $U, V \in \mathcal{T}_{0}^{\mathbf{p}}(\lambda)$. Then by (1.8.2) and the definition of the bilinear form on $W^{\lambda^{[k]}}$, we have

$$
\begin{aligned}
\bar{\varphi}_{U S} \bar{\varphi}_{T V} & =\left(\varphi_{U^{[1]} S^{[1]}} \otimes \cdots \otimes \varphi_{U^{[g] ~} S^{[g]}}\right)\left(\varphi_{T^{[1]} V^{[1]}} \otimes \cdots \otimes \varphi_{T^{[g]} V^{[g]}}\right) \\
& =\varphi_{U^{[1]} S^{[1]}} \varphi_{T^{[1]} V^{[1]}} \otimes \cdots \otimes \varphi_{U^{[g]} S^{[g]}} \varphi_{T^{[g]} V^{[g]}} \\
& \equiv\left\langle\varphi_{S^{[1]}}, \varphi_{T^{[1]}}\right\rangle \varphi_{U^{[1]} V^{[1]}} \otimes \otimes\left\langle\varphi_{S^{[g]}}, \varphi_{T^{[g]}}\right\rangle \varphi_{U^{[g]} V^{[g]}} \\
& \quad \bmod \mathscr{S}\left(\Lambda_{n_{1}}\right)^{\lambda^{[1]}} \otimes \cdots \otimes \mathscr{S}\left(\Lambda_{n_{k}}\right)^{\vee \lambda^{[g]}} \\
& =\left\langle\varphi_{S^{[1]}}, \varphi_{T^{[1]}}\right\rangle \cdots\left\langle\varphi_{S^{[g]}}, \varphi_{T^{[g]}}\right\rangle \varphi_{U^{[1]} V^{[1]}} \otimes \cdots \otimes \varphi_{U^{[g]} V^{[g]}} \\
& =\left\langle\varphi_{S^{[1]}}, \varphi_{T^{[1]}}\right\rangle\left\langle\varphi_{S^{[g]}}, \varphi_{T^{[g]}}\right\rangle \bar{\varphi}_{U^{\prime}} .
\end{aligned}
$$

Since $\mathscr{S}\left(\Lambda_{n_{1}}\right)^{\vee \lambda^{[1]}} \otimes \cdots \otimes \mathscr{S}\left(\Lambda_{n_{k}}\right)^{\vee \lambda^{[g]}} \subset\left(\overline{\mathscr{S}}^{\mathrm{p}}\right)^{\vee \lambda}$, we see that

$$
\left\langle\bar{\varphi}_{S}, \bar{\varphi}_{T}\right\rangle_{\mathbf{p}} \bar{\varphi}_{U V} \equiv \bar{\varphi}_{U S} \bar{\varphi}_{T V} \equiv\left\langle\varphi_{S^{[1]}}, \varphi_{T^{[1]}}\right\rangle \cdots\left\langle\varphi_{S^{[g]}}, \varphi_{T^{[g]}}\right\rangle \bar{\varphi}_{U V} \quad \bmod \left(\overline{\mathscr{S}}^{\mathbf{p}}\right)^{\vee \lambda} \text {. }
$$

The lemma is proved.

Remark 1.10. For the isomorphism in (1.8.3), we do not need to assume that $R$ is a field; for (1.8.4) and (1.8.5), we need that $R$ is a field.

Theorem 1.11 ([SW, Theorem 4.17]). Assume that $R$ is a field. For $\lambda, \mu \in \Lambda^{+}$ such that $\alpha_{\mathbf{p}}(\lambda)=\alpha_{\mathbf{p}}(\mu)$, we have the following:

$$
d_{\lambda \mu}=\bar{d}_{\lambda \mu}=\prod_{k=1}^{g} d_{\lambda^{[k]} \mu^{[k]}},
$$

where $d_{\lambda^{[k]} \mu^{[k]}}=\left[W^{\lambda^{[k]}}: L^{\mu^{[k]}}\right]$.

\section{Decomposition numbers With Jantzen filtration}

2.1. Throughout the rest of this paper, we assume that $R$ is a discrete valuation ring. Let $\wp$ be a unique maximal ideal of $R$ and $F=R / \wp$ the residue field. Fix $\widehat{q}, \widehat{Q}_{1}, \cdots, \widehat{Q}_{r}$ in $R$ and let $q=\widehat{q}+\wp, Q_{1}=\widehat{Q}_{1}+\wp, \cdots, Q_{r}=\widehat{Q}_{r}+\wp$ be their canonical images in $F$. Moreover, let $K$ be the quotient field of $R$. Then $(K, R, F)$ is a modular system with parameters. Let $\mathscr{S}_{R}=\mathscr{S}_{R}(\Lambda)$ be the cyclotomic $\widehat{q}$ Schur algebra over $R$ with parameters $\widehat{q}, \widehat{Q}_{1}, \cdots, \widehat{Q}_{r}$ and let $\mathscr{S}=\mathscr{S}(\Lambda)$ be the 
cyclotomic $q$-Schur algebra over $F$ with parameters $q, Q_{1}, \cdots, Q_{r}$. Then $\mathscr{S}=$ $\left(\mathscr{S}_{R}+\wp \mathscr{S}_{R}\right) / \wp \mathscr{S}_{R}$.

We consider the subalgebra $\mathscr{S}_{R}^{\mathbf{p}}$ (resp. $\mathscr{S}^{\mathbf{p}}$ ) of $\mathscr{S}_{R}$ (resp. $\mathscr{S}$ ) and its quotient $\overline{\mathscr{S}}_{R}^{\mathbf{p}}$ (resp. $\overline{\mathscr{S}}^{\mathbf{p}}$ ) as in the previous section with the notation there. Note that the subscript $R$ is used to indicate the objects related to $R$.

For $\lambda \in \Lambda^{+}$, let $W_{R}^{\lambda}$ be the Weyl module of $\mathscr{S}_{R}$. For $i \in \mathbb{Z}_{\geq 0}$, we set

$$
W_{R}^{\lambda}(i)=\left\{x \in W_{R}^{\lambda} \mid\langle x, y\rangle \in \wp^{i} \text { for any } y \in W_{R}^{\lambda}\right\}
$$

and define

$$
W^{\lambda}(i)=\left(W_{R}^{\lambda}(i)+\wp W_{R}^{\lambda}\right) / \wp W_{R}^{\lambda}
$$

Then $W^{\lambda}=W^{\lambda}(0)$ is the Weyl module of $\mathscr{S}$, and we have the Jantzen filtration of $W^{\lambda}$,

$$
W^{\lambda}=W^{\lambda}(0) \supset W^{\lambda}(1) \supset W^{\lambda}(2) \supset \cdots .
$$

Similarly, by using the bilinear form $\langle,\rangle_{\mathbf{p}}$ on $\bar{Z}_{R}^{\lambda}$, one can define the Jantzen filtration of the $\overline{\mathscr{S}}^{\mathbf{p}}$-module $\bar{Z}^{\lambda}$,

$$
\bar{Z}^{\lambda}=\bar{Z}^{\lambda}(0) \supset \bar{Z}^{\lambda}(1) \supset \bar{Z}^{\lambda}(2) \supset \cdots .
$$

Moreover, for the Weyl module $Z_{R}^{(\lambda, 0)}$ of $\mathscr{S}_{R}^{\mathbf{p}}$, we set

$$
Z_{R}^{(\lambda, 0)}(i)=\left\{x \in Z_{R}^{(\lambda, 0)} \mid \beta_{\lambda}(y, x) \in \wp^{i} \text { for any } y \in \diamond Z_{R}^{(\lambda, 0)}\right\}
$$

and define

$$
Z^{(\lambda, 0)}(i)=\left(Z_{R}^{(\lambda, 0)}(i)+\wp Z_{R}^{(\lambda, 0)}\right) / \wp Z_{R}^{(\lambda, 0)}
$$

Then we have the Jantzen filtration of $Z^{(\lambda, 0)}$ :

$$
Z^{(\lambda, 0)}=Z^{(\lambda, 0)}(0) \supset Z^{(\lambda, 0)}(1) \supset Z^{(\lambda, 0)}(2) \supset \cdots .
$$

Since $W^{\lambda}$ is a finite dimentional $F$-vector space, one can find a positive integer $k$ such that $W^{\lambda}\left(k^{\prime}\right)=W^{\lambda}(k)$ for any $k^{\prime}>k$. We choose a minimal $k$ in such numbers and set $W^{\lambda}(k+1)=0$. Then the Jantzen filtration of $W^{\lambda}$ becomes a finite sequence. Similarly, Jantzen filtrations of $Z^{(\lambda, 0)}$ and $\bar{Z}^{\lambda}$ also become finite sequences.

We can easily see that $W^{\lambda}(i)$ (resp. $Z^{(\lambda, 0)}(i), \bar{Z}^{\lambda}(i)$ ) is a $\mathscr{S}$-submodule of $W^{\lambda}$ (resp. $\mathscr{S}^{\mathbf{p}}$-submodule of $Z^{(\lambda, 0)}, \overline{\mathscr{S}}^{\mathbf{p}}$-submodule of $\bar{Z}^{\lambda}$ ) by associativity of the bilinear form (1.3.1) (resp. (1.4.1), (1.5.1) ).

2.2. Take $\lambda, \mu \in \Lambda^{+}$, and let $W^{\lambda}=W^{\lambda}(0) \supset W^{\lambda}(1) \supset \cdots$ be the Jantzen filtration of $W^{\lambda}$. Let $\left[W^{\lambda}(i) / W^{\lambda}(i+1): L^{\mu}\right]$ be the composition multiplicity of $L^{\mu}$ in $W^{\lambda}(i) / W^{\lambda}(i+1)$. Let $v$ be an indeterminate. We define a polynomial $d_{\lambda \mu}(v)$ by

$$
d_{\lambda \mu}(v)=\sum_{i \geq 0}\left[W^{\lambda}(i) / W^{\lambda}(i+1): L^{\mu}\right] \cdot v^{i} .
$$

Similarly we define, for $Z^{(\lambda, 0)}$ and $\bar{Z}^{\lambda}$,

$$
\begin{gathered}
d_{\lambda \mu}^{(\lambda, 0)}(v)=\sum_{i \geq 0}\left[Z^{(\lambda, 0)}(i) / Z^{(\lambda, 0)}(i+1): L^{(\mu, 0)}\right] \cdot v^{i}, \\
\bar{d}_{\lambda \mu}(v)=\sum_{i \geq 0}\left[\bar{Z}^{\lambda}(i) / \bar{Z}^{\lambda}(i+1): \bar{L}^{\mu}\right] \cdot v^{i} .
\end{gathered}
$$


Thus $d_{\lambda \mu}(v), d_{\lambda \mu}^{(\lambda, 0)}(v)$ and $\bar{d}_{\lambda \mu}(v)$ are porynomials whose coefficients are nonnegative integers. Note that since the Jantzen filtrations of $W^{\lambda}$, etc. are finite sequences, these summations are finite sums. We call $d_{\lambda \mu}(v)\left(\operatorname{resp} . d_{\lambda \mu}^{(\lambda, 0)}(v), \bar{d}_{\lambda \mu}(v)\right)$

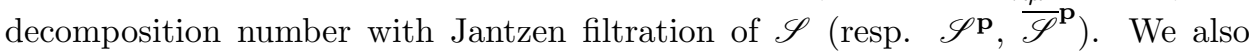
call them $v$-decomposition numbers as they coincide at $v=1$ with decomposition numbers given in 1.6 .

We have the following relation between $d_{\lambda \mu}^{(\lambda, 0)}(v)$ and $\bar{d}_{\lambda \mu}(v)$.

Proposition 2.3. For $\lambda, \mu \in \Lambda$, we have:

(i) If $\alpha_{\mathbf{p}}(\lambda) \neq \alpha_{\mathbf{p}}(\mu)$, then $\bar{d}_{\lambda \mu}(v)=d_{\lambda \mu}^{(\lambda, 0)}(v)=0$.

(ii) $\left[\bar{Z}^{\lambda}(i) / \bar{Z}^{\lambda}(i+1): \bar{L}^{\mu}\right]=\left[Z^{(\lambda, 0)}(i) / Z^{(\lambda, 0)}(i+1): L^{(\mu, 0)}\right]$ for any $i \geq 0$. Hence we have $\bar{d}_{\lambda \mu}(v)=d_{\lambda \mu}^{(\lambda, 0)}(v)$.

Proof. (ii) is clear since $d_{\lambda \mu}^{(\lambda, 0)}=\bar{d}_{\lambda \mu}=0$ by [SW, Proposition 3.3].

Recall that $\bar{Z}^{\lambda} \cong Z^{(\lambda, 0)}$ and $\bar{L}^{\mu} \cong L^{(\mu, 0)}$ as $\mathscr{S}^{\mathbf{p}}$-modules by [SW, Lemma 3.2]. By definition, we have $\beta_{\lambda}\left(\varphi_{S}^{(\lambda, 0)}, \varphi_{T}^{(\lambda, 0)}\right)=\left\langle\bar{\varphi}_{T}, \bar{\varphi}_{S}\right\rangle_{\mathbf{p}}$ for any $S, T \in \mathcal{T}_{0}^{\mathbf{p}}(\lambda)$. Then under the isomorphism $\bar{Z}^{\lambda} \cong Z^{(\lambda, 0)}$, the Jantzen filtration of $\bar{Z}^{\lambda}$ coincides with that of $Z^{(\lambda, 0)}$. So (iil) is proved.

2.4. Next, we consider the relation between $d_{\lambda \mu}^{(\lambda, 0)}(v)$ and $d_{\lambda \mu}(v)$. In order to see this we prepare two lemmas. Recall that there exists an injective $\mathscr{S}^{\mathbf{p}}$-homomorphism $f_{\lambda}: Z^{(\lambda, 0)} \hookrightarrow W^{\lambda}$ such that $f_{\lambda}\left(\varphi_{T}^{(\lambda, 0)}\right)=\varphi_{T}$ for $T \in \mathcal{T}_{0}^{\mathbf{p}}(\lambda)$ by [SW Lemma 3.5] and that $Z^{(\lambda, 0)} \otimes_{\mathscr{S} \mathbf{p}} \mathscr{S} \cong W^{\lambda}$ as an $\mathscr{S}$-module by [SW, Proposition 3.6]. Let $\iota_{i}: Z^{(\lambda, 0)}(i) \hookrightarrow Z^{(\lambda, 0)}$ be an inclusion map. Then $\left(\iota_{i} \otimes \operatorname{id} \mathscr{S}\right)\left(Z^{(\lambda, 0)}(i) \otimes \mathscr{S} \mathbf{p} \mathscr{S}\right)$ is the $\mathscr{S}$-submodule of $Z^{(\lambda, 0)} \otimes \mathscr{S} \mathbf{p} \mathscr{S}$. Similar results hold also for $R$. We have the following.

Lemma 2.5. Let $\lambda \in \Lambda^{+}$. For any $i \geq 0$, we have

$$
f_{\lambda}^{-1}\left(W^{\lambda}(i)\right)=Z^{(\lambda, 0)}(i)
$$

Proof. By definition, we see that $\beta\left(\varphi_{T}^{(\lambda, 0)}, \varphi_{S}^{(\lambda, 0)}\right)=\left\langle\varphi_{S}, \varphi_{T}\right\rangle$ for any $S, T \in \mathcal{T}_{0}^{\mathbf{p}}(\lambda)$, and that $\left\langle\varphi_{S}, \varphi_{T}\right\rangle=0$ if $S \in \mathcal{T}_{0}^{\mathbf{p}}(\lambda), T \in \mathcal{T}_{0}(\lambda) \backslash \mathcal{T}_{0}^{\mathbf{p}}(\lambda)$. Then for $x \in Z_{R}^{(\lambda, 0)}$, we have

$$
\begin{aligned}
x \in Z_{R}^{(\lambda, 0)}(i) & \Leftrightarrow \beta_{\lambda}\left(\varphi_{T}^{(\lambda, 0)}, x\right) \in \wp^{i} \quad \text { for any } T \in \mathcal{T}_{0}^{\mathbf{p}}(\lambda) \\
& \Leftrightarrow\left\langle f_{\lambda}(x), \varphi_{T}\right\rangle \in \wp^{i} \quad \text { for any } T \in \mathcal{T}_{0}(\lambda) \\
& \Leftrightarrow f_{\lambda}(x) \in W_{R}^{\lambda}(i) .
\end{aligned}
$$

By taking the quotient, we obtain the lemma.

Lemma 2.6. Let $\lambda \in \Lambda^{+}$. For any $i \geq 0$, we have

$$
\left(\iota_{i} \otimes \operatorname{id} \mathscr{S}\right)\left(Z^{(\lambda, 0)}(i) \otimes \mathscr{S} \mathbf{p} \mathscr{S}\right) \subset W^{\lambda}(i)
$$

under the isomorphism $Z^{(\lambda, 0)} \otimes \mathscr{S} \mathbf{p} \mathscr{S} \cong W^{\lambda}$.

Proof. Recall that any element of $Z_{R}^{(\lambda, 0)}$ can be written in the form $\varphi_{T^{\lambda}}^{(\lambda, 0)} \cdot \psi$ with $\psi \in$ $\mathscr{S}_{R}^{\mathbf{p}}$. Moreover, it follows from [SW] Proposition 3.6] that, under the isomorphism $g_{\lambda}: Z_{R}^{(\lambda, 0)} \otimes \mathscr{S}_{\mathbf{p}} \mathscr{S}_{R} \stackrel{\sim}{\longrightarrow} W_{R}^{\lambda}$, we have $g_{\lambda}\left(\varphi_{T^{\lambda}}^{(\lambda, 0)} \cdot \psi \otimes \varphi\right)=\varphi_{T^{\lambda}} \cdot \psi \varphi$ for $\psi \in \mathscr{S}_{R}^{\mathbf{p}}, \varphi \in$ 
$\mathscr{S}_{R}$. This is true also for $Z^{(\lambda, 0)}$ and $W^{\lambda}$. Thus in order to show the lemma, it is enough to prove the following.

(2.6.1) Suppose that $\varphi_{T^{\lambda}}^{(\lambda, 0)} \psi \in Z^{(\lambda, 0)}(i)$ for $\psi \in \mathscr{S}^{\mathbf{p}}$. Then we have $\varphi_{T^{\lambda}} \psi \varphi \in$ $W^{\lambda}(i)$ for any $\varphi \in \mathscr{S}$.

Now take $\varphi_{T^{\lambda}}^{(\lambda, 0)} \psi \in Z_{R}^{(\lambda, 0)}$. If $\varphi_{T^{\lambda}}^{(\lambda, 0)} \cdot \psi \in Z_{R}^{(\lambda, 0)}(i)$, then $\beta_{\lambda}\left(x, \varphi_{T^{\lambda}}^{(\lambda, 0)} \psi\right) \in \wp^{i}$ for any $x \in \diamond Z_{R}^{(\lambda, 0)}$. This implies that $\left\langle\varphi_{T^{\lambda}} \psi, y\right\rangle \in \wp^{i}$ for any $y \in W_{R}^{\lambda}$ by a similar argument as in the proof of Lemma 2.5

Since $\left\langle\varphi_{T^{\lambda}} \psi \varphi, y\right\rangle=\left\langle\varphi_{T^{\lambda}} \psi, y \varphi^{*}\right\rangle$ for any $y \in W_{R}^{\lambda}$ and any $\varphi \in \mathscr{S}_{R}$, we see that $\varphi_{T^{\lambda}}^{(\lambda, 0)} \psi \in Z_{R}^{(\lambda, 0)}(i)$ implies that $\varphi_{T^{\lambda}} \psi \varphi \in W_{R}^{\lambda}(i)$. By taking the quotient, we obtain (2.6.1). Thus the lemma is proved.

These two lemmas imply the following proposition about the relation between $d_{\lambda \mu}^{(\lambda, 0)}(v)$ and $d_{\lambda \mu}(v)$.

Proposition 2.7. Let $\lambda, \mu \in \Lambda^{+}$be such that $\alpha_{\mathbf{p}}(\lambda)=\alpha_{\mathbf{p}}(\mu)$. Then for any $i \geq 0$, we have

$$
\left[Z^{(\lambda, 0)}(i) / Z^{(\lambda, 0)}(i+1): L^{(\mu, 0)}\right]=\left[W^{\lambda}(i) / W^{\lambda}(i+1): L^{\mu}\right] .
$$

Hence we have $d_{\lambda \mu}^{(\lambda, 0)}(v)=d_{\lambda \mu}(v)$ if $\alpha_{\mathbf{p}}(\lambda)=\alpha_{\mathbf{p}}(\mu)$.

Proof. Fix $\lambda, \mu \in \Lambda^{+}$such that $\alpha_{\mathbf{p}}(\lambda)=\alpha_{\mathbf{p}}(\mu)$, and an integer $i \geq 0$. Thanks to Lemma 2.5. we have the following result by similar arguments as in the proof of SW, Proposition 3.12]:

$$
\left[Z^{(\lambda, 0)}(i) / Z^{(\lambda, 0)}(i+1): L^{(\mu, 0)}\right] \geq\left[W^{\lambda}(i) / W^{\lambda}(i+1): L^{\mu}\right] .
$$

Conversly, thanks to Lemma 2.6, we have the following result by similar arguments as in the proof of [SW, Proposition 3.11]:

$$
\left[Z^{(\lambda, 0)}(i): L^{(\mu, 0)}\right] \leq\left[W^{\lambda}(i): L^{\mu}\right] .
$$

We remark that this does not imply directly

$$
\left[Z^{(\lambda, 0)}(i) / Z^{(\lambda, 0)}(i+1): L^{(\mu, 0)}\right] \leq\left[W^{\lambda}(i) / W^{\lambda}(i+1): L^{\mu}\right]
$$

since we cannot see whether $\left(\iota_{i+1} \otimes \operatorname{id} \mathscr{S}\right)\left(Z^{(\lambda, 0)}(i+1) \otimes \mathscr{S}_{\mathbf{p}} \mathscr{S}\right)=W^{\lambda}(i+1)$ or not. Instead, we argue as follows. Let

$$
\begin{gathered}
W^{\lambda}=W^{\lambda}(0) \supset W^{\lambda}(1) \supset \cdots \supset W^{\lambda}(k) \supsetneqq W^{\lambda}(k+1)=0, \\
Z^{(\lambda, 0)}=Z^{(\lambda, 0)}(0) \supset Z^{(\lambda, 0)}(1) \supset \cdots \supset Z^{(\lambda, 0)}(l) \supsetneqq Z^{(\lambda, 0)}(l+1)=0,
\end{gathered}
$$

be the Jantzen filtrations of $W^{\lambda}$ and $Z^{(\lambda, 0)}$ respectively. Then we have

$$
\left(\iota_{k+1} \otimes \operatorname{id}_{\mathscr{S}}\right)\left(Z^{(\lambda, 0)}(k+1) \otimes_{\mathscr{S} \mathbf{p}} \mathscr{S}\right) \subset W^{\lambda}(k+1)=0
$$

by Lemma 2.6. This implies that $Z^{(\lambda, 0)}(k+1)=0$ since $(\iota \otimes \operatorname{id} \mathscr{S})\left(M \otimes_{\mathscr{S}}^{\mathbf{p}} \mathscr{S}\right) \neq 0$ for any non-zero submodule $M$ of $Z^{(\lambda, 0)}$ and the inclusion map $\iota: M \hookrightarrow Z^{(\lambda, 0)}$ by [SW, Lemma 3.8 (ii)]. So we have $l \leqq k$.

Now, if $L^{\mu}$ is a composition factor of $W^{\lambda}(i) / W^{\lambda}(i+1)$, then we have

$$
1 \leq\left[W^{\lambda}(i) / W^{\lambda}(i+1): L^{\mu}\right] \leq\left[Z^{(\lambda, 0)}(i) / Z^{(\lambda, 0)}(i+1): L^{(\mu, 0)}\right]
$$


by (2.7.1). Hence, we have $Z^{(\lambda, 0)}(i) \neq 0$. This implies that $i \leqq l$ if $L^{\mu}$ is a composition factor of $W^{\lambda}(i)$. In particular, $\left[W^{\lambda}(l+1): L^{\mu}\right]=0$. Thus we have

$$
\begin{gathered}
{\left[Z^{(\lambda, 0)}(l) / Z^{(\lambda, 0)}(l+1): L^{(\mu, 0)}\right]=\left[Z^{(\lambda, 0)}(l): L^{(\mu, 0)}\right],} \\
{\left[W^{\lambda}(l) / W^{\lambda}(l+1): L^{\mu}\right]=\left[W^{\lambda}(l): L^{\mu}\right] .}
\end{gathered}
$$

Combining these equalities with (2.7.1) and (2.7.2), we have

$$
\left[Z^{(\lambda, 0)}(l) / Z^{(\lambda, 0)}(l+1): L^{(\mu, 0)}\right]=\left[W^{\lambda}(l) / W^{\lambda}(l+1): L^{\mu}\right],
$$

and so

$$
\left[Z^{(\lambda, 0)}(l): L^{(\mu, 0)}\right]=\left[W^{\lambda}(l): L^{\mu}\right] .
$$

Next we consider the case where $i=l-1$. Note that

$$
\begin{gathered}
{\left[Z^{(\lambda, 0)}(l-1) / Z^{(\lambda, 0)}(l): L^{(\mu, 0)}\right]=\left[Z^{(\lambda, 0)}(l-1): L^{(\mu, 0)}\right]-\left[Z^{(\lambda, 0)}(l): L^{(\mu, 0)}\right],} \\
{\left[W^{\lambda}(l-1) / W^{\lambda}(l): L^{\mu}\right]=\left[W^{\lambda}(l-1): L^{\mu}\right]-\left[W^{\lambda}(l): L^{\mu}\right] .}
\end{gathered}
$$

Combined with (2.7.1), (2.7.2) and (2.7.3), we have

$$
\left[Z^{(\lambda, 0)}(l-1) / Z^{(\lambda, 0)}(l): L^{(\mu, 0)}\right]=\left[W^{\lambda}(l-1) / W^{\lambda}(l): L^{\mu}\right]
$$

and so $\left[Z^{(\lambda, 0)}(l-1): L^{(\mu, 0)}\right]=\left[W^{\lambda}(l-1): L^{\mu}\right]$. Therefore by backward induction on $l$, we obtain the proposition.

Combining Proposition 2.3 and Proposition 2.7, we have the following theorem.

Theorem 2.8. For any $\lambda, \mu \in \Lambda^{+}$such that $\alpha_{\mathbf{p}}(\lambda)=\alpha_{\mathbf{p}}(\mu)$, we have

$$
\bar{d}_{\lambda \mu}(v)=d_{\lambda \mu}^{(\lambda, 0)}(v)=d_{\lambda \mu}(v) .
$$

If we specialize $v=1$, the theorem reduces to Theorem 1.7 .

2.9. For later use, we shall consider the basis of $W_{R}^{\lambda}(i)$, following the computation in the proof of [M2, Lemma 5.30]. Let $\pi$ be the generator of $\wp$, namely $\wp=(\pi)$, and $\nu_{\wp}$ be the valuation map on $R$. Let $G^{\lambda}=\left(\left\langle\varphi_{S}, \varphi_{T}\right\rangle\right)_{S, T \in \mathcal{T}_{0}(\lambda)}$ be the Gram matrix of $W_{R}^{\lambda}$. Since $R$ is a PID, there exist $P, Q \in \mathrm{GL}_{N}(R)$ (where $N=\left|\mathcal{T}_{0}(\lambda)\right|$ ) such that $P G^{\lambda} Q=\operatorname{diag}\left(d_{S_{1}}, d_{S_{2}}, \cdots, d_{S_{N}}\right)$, where $d_{S_{k}} \in R$ and $\left\{S_{1}, \cdots, S_{N}\right\}=\mathcal{T}_{0}(\lambda)$. Let $P=\left(p_{S T}\right)_{S, T \in \mathcal{T}_{0}(\lambda)}, Q=\left(q_{S T}\right)_{S, T \in \mathcal{T}_{0}(\lambda)}$ and we define, for $S, T \in \mathcal{T}_{0}(\lambda)$,

$$
f_{S}=\sum_{S^{\prime} \in \mathcal{T}_{0}(\lambda)} p_{S S^{\prime}} \varphi_{S^{\prime}}, \quad g_{T}=\sum_{T^{\prime} \in \mathcal{T}_{0}(\lambda)} q_{T^{\prime} T} \varphi_{T^{\prime}} .
$$

Since both $P$ and $Q$ are regular matrices, $\left\{f_{S} \mid S \in \mathcal{T}_{0}(\lambda)\right\}$ and $\left\{g_{T} \mid T \in \mathcal{T}_{0}(\lambda)\right\}$ are basis of $W_{R}^{\lambda}$, respectively. Moreover, we have $\operatorname{diag}\left(d_{S_{1}}, \cdots, d_{S_{N}}\right)=P G^{\lambda} Q=$ $\left(\left\langle f_{S}, g_{T}\right\rangle\right)_{S, T \in \mathcal{T}_{0}(\lambda)}$ by definition. Thus we have

$$
\left\langle f_{S}, g_{T}\right\rangle=\delta_{S T} d_{S} \quad\left(S, T \in \mathcal{T}_{0}(\lambda)\right)
$$

where $\delta_{S T}=1$ if $S=T$ and $\delta_{S T}=0$ otherwise. For $x=\sum_{S \in \mathcal{T}_{0}(\lambda)} r_{S} f_{S} \in$ $W_{R}^{\lambda} \quad\left(r_{S} \in R\right)$, we have

$$
\begin{aligned}
x \in W_{R}^{\lambda}(i) & \Leftrightarrow\left\langle x, g_{T}\right\rangle \in \wp^{i} \quad \text { for any } T \in \mathcal{T}_{0}(\lambda) \\
& \Leftrightarrow r_{T} d_{T} \in \wp^{i} \quad \text { for any } T \in \mathcal{T}_{0}(\lambda) \quad(\text { by }(2.9 .1)) \\
& \Leftrightarrow \nu_{\wp}\left(r_{T} d_{T}\right)=\nu_{\wp}\left(r_{T}\right)+\nu_{\wp}\left(d_{T}\right) \geq i \quad \text { for any } T \in \mathcal{T}_{0}(\lambda) .
\end{aligned}
$$


It follows from this that $W_{R}^{\lambda}(i)$ is a free $R$-module with basis

$$
\left\{f_{T} \mid T \in \mathcal{T}_{0}(\lambda), \nu_{\wp}\left(d_{T}\right) \geq i\right\} \cup\left\{\pi^{i-\nu_{\wp}\left(d_{T}\right)} f_{T} \mid T \in \mathcal{T}_{0}(\lambda), \nu_{\wp}\left(d_{T}\right)<i\right\} .
$$

2.10. We consider the Jantzen filtration of $W^{\lambda[k]}(1 \leq k \leq g)$ as in the case of $W^{\lambda}$ and use the notation similar to the case of $W^{\lambda}$. Since we see that $W_{R}^{\lambda^{[k]}}\left(i_{k}\right)$ $\left(i_{k} \geq 0\right)$ is a free $R$-module (see 2.9) $), W_{R}^{\lambda^{[1]}}\left(i_{1}\right) \otimes \cdots \otimes W_{R}^{\lambda^{[g]}}\left(i_{g}\right)\left(\left(i_{1}, \cdots, i_{g}\right) \in Z_{\geq 0}^{g}\right)$ becomes the submodule of $W_{R}^{\lambda^{[1]}} \otimes \cdots \otimes W_{R}^{\lambda^{[g]}}$.

For $1 \leq k \leq g$, let $\left\{f_{S^{[k]}} \mid S^{[k]} \in \mathcal{T}_{0}\left(\lambda^{[k]}\right)\right\}$ and $\left\{g_{T^{[k]}} \mid T^{[k]} \in \mathcal{T}_{0}\left(\lambda^{[k]}\right)\right\}$ be the bases of $W_{R}^{\lambda^{[k]}}$ as of $W_{R}^{\lambda}$ in 2.9, For $S, T \in \mathcal{T}_{0}^{\mathbf{p}}(\lambda)$, we define $\bar{f}_{S}:=f_{S^{[1]}} \otimes \cdots \otimes f_{S^{[g]}}$ and $\bar{g}_{T}:=g_{T^{[1]}} \otimes \cdots \otimes g_{T^{[g]}}$. Then $\left\{\bar{f}_{S} \mid S \in \mathcal{T}_{0}^{\mathbf{p}}(\lambda)\right\}$ and $\left\{\bar{g}_{T} \mid T \in \mathcal{T}_{0}^{\mathbf{p}}(\lambda)\right\}$ turn out to be the bases of $\bar{Z}_{R}^{\lambda}$. By Lemma 1.9 and (2.9.1), we have

$$
\left\langle\bar{f}_{S}, \bar{g}_{T}\right\rangle_{\mathbf{p}}=\delta_{S T} d_{T^{[1]}} \cdots d_{T^{[g]}} \quad \text { for } S, T \in \mathcal{T}_{0}^{\mathbf{p}}(\lambda) .
$$

We set $d_{T}=d_{T^{[1]}} \cdots d_{\left.T^{[g]}\right]}$. Then we have the following result by a similar argument as in 2.9, $\bar{Z}_{R}^{\lambda}(i)$ is a free $R$-module with basis

$$
\left\{\bar{f}_{T} \mid T \in \mathcal{T}_{0}^{\mathbf{p}}(\lambda), \nu_{\wp}\left(d_{T}\right) \geq i\right\} \cup\left\{\pi^{i-\nu_{\wp}\left(d_{T}\right)} \bar{f}_{T} \mid T \in \mathcal{T}_{0}^{\mathbf{p}}(\lambda), \nu_{\wp}\left(d_{T}\right)<i\right\} .
$$

Recall that $\Delta_{i, g}$ is the set of $\left(i_{1}, \cdots, i_{g}\right) \in \mathbb{Z}_{\geq 0}^{g}$ such that $i_{1}+\cdots+i_{g}=i$. Then we have the following proposition.

Proposition 2.11. Let $\lambda \in \Lambda^{+}$and $i \geq 0$. Under the isomorphism $\bar{Z}_{R}^{\lambda} \cong W_{R}^{\lambda^{[1]}} \otimes$ $\cdots \otimes W_{R}^{\lambda^{[g]}}$, we have

$$
\bar{Z}_{R}^{\lambda}(i)=\sum_{\left(i_{1}, \cdots, i_{g}\right) \in \Delta_{i, g}} W_{R}^{\lambda^{[1]}}\left(i_{1}\right) \otimes \cdots \otimes W_{R}^{\lambda^{[g]}}\left(i_{g}\right) .
$$

Proof. First we show that the right-hand side is contained in the left-hand side. Take $x=x^{[1]} \otimes \cdots \otimes x^{[g]} \in W_{R}^{\lambda^{[1]}}\left(i_{1}\right) \otimes \cdots \otimes W_{R}^{\lambda^{[g]}}\left(i_{g}\right)$ such that $i_{1}+\cdots+i_{g}=i$. By Lemma 1.9, we have

$$
\begin{aligned}
\left\langle x, \bar{\varphi}_{T}\right\rangle_{\mathbf{p}} & =\left\langle x^{[1]}, \varphi_{T^{[1]}}\right\rangle \cdots\left\langle x^{[g]}, \varphi_{T^{[g]}}\right\rangle \\
& \in \wp^{i_{1}} \cdots \wp^{i_{g}}=\wp^{i} \quad \text { for any } T \in \mathcal{T}_{0}^{\mathbf{p}}(\lambda) .
\end{aligned}
$$

Thus we have $x \in \bar{Z}_{R}^{\lambda}(i)$.

Then in order to show the equality, we only have to show that the basis element of $\bar{Z}_{R}^{\lambda}(i)$ is contained in the right-hand side of (2.11.1). First, we consider $\bar{f}_{T}$ such that $\nu_{\wp}\left(d_{T}\right) \geq i$. Since $\nu_{\wp}\left(d_{T}\right)=\nu_{\wp}\left(d_{T^{[1]}}\right)+\cdots+\nu_{\wp}\left(d_{T^{[g]}}\right)$, one can find $\left(i_{1}, \cdots, i_{g}\right) \in \mathbb{Z}_{\geq 0}^{g}$ such that $i_{1}+\cdots+i_{g}=i$ and that $\nu_{\wp}\left(d_{T^{[k]}}\right) \geq i_{k}$ for $k=1, \cdots, g$. Then

$$
\bar{f}_{T}=f_{T^{[1]}} \otimes \cdots \otimes f_{T^{[g]}} \in W_{R}^{\lambda^{[1]}}\left(i_{1}\right) \otimes \cdots \otimes W_{R}^{\lambda^{[g]}}\left(i_{g}\right),
$$

and so $\bar{f}_{T}$ is contained in the right-hand side of (2.11.1).

Next we consider $\bar{f}_{T}$ such that $\nu_{\wp}\left(d_{T}\right)<i$. Then one can find $\left(i_{1}, \cdots, i_{g}\right)$ such that $i_{1}+\cdots+i_{g}=i$ and that $\nu_{\wp}\left(d_{T^{[k]}}\right) \leq i_{k}$ for $k=1, \cdots, g$. Therefore $\pi^{i-\nu_{\wp}\left(d_{T}\right)} \bar{f}_{T}=\left(\pi^{i_{1}-\nu_{\wp}\left(d_{T^{[1]}}\right)} f_{T^{[1]}}\right) \otimes \cdots \otimes\left(\pi^{i_{g}-\nu_{\wp}\left(d_{T^{[g]}}\right)} f_{T^{[g]}}\right)$ is also contained in the right-hand side of (2.11.1). The proposition is proved.

We have the following corollary. 
Corollary 2.12. For $\lambda \in \Lambda^{+}$and $i \geq 0$, under the isomorphism $\bar{Z}^{\lambda} \cong W^{\lambda^{[1]}} \otimes$ $\cdots \otimes W^{\lambda^{[g]}}$, we have

$$
\bar{Z}^{\lambda}(i)=\sum_{\left(i_{1}, \cdots, i_{g}\right) \in \Delta_{i, g}} W^{\lambda^{[1]}}\left(i_{1}\right) \otimes \cdots \otimes W^{\lambda^{[g]}}\left(i_{g}\right) .
$$

Proof. By definition, we have

$$
\bar{Z}^{\lambda}(i)=\left(\bar{Z}_{R}^{\lambda}(i)+\wp \bar{Z}_{R}^{\lambda}\right) / \wp \bar{Z}_{R}^{\lambda} \cong \bar{Z}_{R}^{\lambda}(i) /\left(\bar{Z}_{R}^{\lambda}(i) \cap \wp \bar{Z}_{R}^{\lambda}\right)
$$

and

$$
\begin{aligned}
W^{\lambda^{[1]}}\left(i_{1}\right) & \otimes \cdots \otimes W^{\lambda^{[g]}}\left(i_{g}\right) \\
= & \left(W_{R}^{\lambda^{[1]}}\left(i_{1}\right)+\wp W_{R}^{\lambda^{[1]}}\right) / \wp W_{R}^{\lambda^{[1]}} \otimes \cdots \otimes\left(W_{R}^{\lambda^{[g]}}\left(i_{g}\right)+\wp W_{R}^{\lambda^{[g]}}\right) / \wp W_{R}^{\lambda^{[g]}} \\
& \cong W_{R}^{\lambda^{[1]}}\left(i_{1}\right) /\left(W_{R}^{\lambda^{[1]}}\left(i_{1}\right) \cap \wp W_{R}^{\lambda^{[1]}}\right) \otimes \cdots \otimes W_{R}^{\lambda^{[g]}}\left(i_{g}\right) /\left(W_{R}^{\lambda^{[g]}}\left(i_{1}\right) \cap \wp W^{\lambda^{[g]}}\right) .
\end{aligned}
$$

By Proposition 2.11, we have a surjective map

$$
\Phi: \bar{Z}_{R}^{\lambda}(i) \rightarrow \sum_{\left(i_{1}, \cdots, i_{g}\right) \in \Delta_{i, g}} W^{\lambda^{[1]}}\left(i_{1}\right) \otimes \cdots \otimes W^{\lambda^{[g]}}\left(i_{g}\right) .
$$

We claim that $\operatorname{Ker} \Phi=\bar{Z}_{R}^{\lambda}(i) \cap \wp \bar{Z}^{\lambda}$. Then the claim implies the corollary. So we shall show the claim. By definition, it is clear that $\operatorname{Ker} \Phi$ is contained in $\bar{Z}_{R}^{\lambda}(i) \cap \wp \bar{Z}^{\lambda}$. Take $x=\sum_{T \in \mathcal{T}_{0}^{\mathbf{p}}(\lambda)} r_{T} \bar{f}_{T} \in \bar{Z}_{R}^{\lambda}(i) \cap \wp \bar{Z}^{\lambda}$. Then $r_{T} \in \wp$ for any $T \in \mathcal{T}_{0}^{\mathbf{p}}(\lambda)$. If $\nu_{\wp}\left(d_{T}\right) \geq i$, then $\bar{f}_{T}=f_{T^{[1]}} \otimes \cdots \otimes f_{T^{[g]}}$ is contained in $W_{R}^{\lambda^{[1]}}\left(i_{1}\right) \otimes \cdots \otimes W_{R}^{\lambda^{[g]}}\left(i_{g}\right)$ for some $\left(i_{1}, \cdots, i_{g}\right) \in \Delta_{i, g}$ by the proof of Proposition 2.11 So we have $r_{T} \bar{f}_{T} \in \operatorname{Ker} \Phi$ for $T \in \mathcal{T}_{o}^{\mathbf{p}}(\lambda)$ such that $\nu_{\wp}\left(d_{T}\right) \geq i$. If $\nu_{\wp}\left(d_{T}\right)<i$, then $r_{T} \bar{f}_{T}=r_{T}^{\prime} \pi^{i-\nu_{\wp}\left(d_{T}\right)} \bar{f}_{T}$ for some $r_{T}^{\prime} \in R$ since $x \in \bar{Z}_{R}^{\lambda}(i)$. By the proof of Proposition 2.11, for some $\left(i_{1}, \cdots, i_{g}\right) \in \Delta_{i, g}, \pi^{i-\nu_{\wp}\left(d_{T}\right)} \bar{f}_{T}=\left(\pi^{i_{1}-\nu_{\wp}\left(d_{T^{[1]}}\right)} f_{T^{[1]}}\right) \otimes \cdots \otimes\left(\pi^{i_{g}-\nu_{\wp}\left(d_{\left.T^{[g]}\right]}\right.} f_{T^{[g]}}\right)$ is contained in $W_{R}^{\lambda^{[1]}}\left(i_{1}\right) \otimes \cdots \otimes W_{R}^{\lambda^{[g]}}\left(i_{g}\right)$. Moreover, one can find at least one $k$ such that $\nu_{\wp}\left(d_{T^{[k]}}\right)<i_{k}$. Then the image of $\pi^{i_{k}-\nu_{\wp}\left(d_{T^{[k]}}\right)} f_{T^{[k]}}$ in $W_{R}^{\lambda^{[k]}}\left(i_{k}\right) /\left(W_{R}^{\lambda^{[k]}}\left(i_{k}\right) \cap\right.$ $\left.\wp W^{\lambda^{[k]}}\right)$ is zero. Hence for $T \in \mathcal{T}_{0}^{\mathbf{p}}$ such that $\nu_{\wp}\left(d_{T}\right)<i, r_{T} \bar{f}_{T}$ is also contained in $\operatorname{Ker} \Phi$. Now the claim is proved, and the corollary follows.

By using the corollary, we show the following lemma.

Lemma 2.13. Let $\lambda, \mu \in \Lambda^{+}$be such that $\alpha_{\mathbf{p}}(\lambda)=\alpha_{\mathbf{p}}(\mu)$. For any $i \geq 0$, we have

$$
\left[\bar{Z}^{\lambda}(i) / \bar{Z}^{\lambda}(i+1): \bar{L}^{\mu}\right]=\sum_{\left(i_{1}, \cdots, i_{g}\right) \in \Delta_{i, g}} \prod_{k=1}^{g}\left[W^{\lambda^{[k]}}\left(i_{k}\right) / W^{\lambda^{[k]}}\left(i_{k+1}\right): L^{\mu^{[k]}}\right] .
$$

Proof. By Corollary 2.12, we have

$$
\begin{aligned}
& \bar{Z}^{\lambda}(i) / \bar{Z}^{\lambda}(i+1)=\left(\sum_{\left(i_{1}, \cdots, i_{g}\right) \in \Delta_{i, g}} W^{\lambda^{[1]}}\left(i_{1}\right) \otimes \cdots \otimes W^{\lambda^{[g]}}\left(i_{g}\right)\right) / \bar{Z}^{\lambda}(i+1) \\
& =\sum_{\left(i_{1}, \cdots, i_{g}\right) \in \Delta_{i, g}}\left(W^{\lambda^{[1]}}\left(i_{1}\right) \otimes \cdots \otimes W^{\lambda^{[g]}}\left(i_{g}\right)\right) /\left(\bar{Z}^{\lambda}(i+1) \cap\left(W^{\lambda^{[1]}}\left(i_{1}\right) \otimes \cdots \otimes W^{\lambda^{[g]}}\left(i_{g}\right)\right)\right) .
\end{aligned}
$$


If $\left(i_{1}, \cdots, i_{g}\right) \neq\left(j_{1}, \cdots, j_{g}\right)$ such that $i_{1}+\cdots+i_{g}=j_{1}+\cdots+j_{g}=i$, then

$$
\begin{array}{r}
\left(W^{\lambda^{[1]}}\left(i_{1}\right) \otimes \cdots \otimes W^{\lambda^{[g]}}\left(i_{g}\right)\right) \cap\left(W^{\lambda^{[1]}}\left(j_{1}\right) \otimes \cdots \otimes W^{\lambda^{[g]}}\left(j_{g}\right)\right) \\
\subset W^{\lambda^{[1]}}\left(k_{1}\right) \otimes \cdots \otimes W^{\lambda^{[g]}}\left(k_{g}\right),
\end{array}
$$

where $k_{l}=\max \left\{i_{l}, j_{l}\right\}$. Since $\left(i_{1}, \cdots, i_{g}\right) \neq\left(j_{1}, \cdots, j_{g}\right), k_{1}+\cdots+k_{g} \geq i+1$. Hence we have

$$
\left(W^{\lambda^{[1]}}\left(i_{1}\right) \otimes \cdots \otimes W^{\lambda^{[g]}}\left(i_{g}\right)\right) \cap\left(W^{\lambda^{[1]}}\left(j_{1}\right) \otimes \cdots \otimes W^{\lambda^{[g]}}\left(j_{g}\right)\right) \subset \bar{Z}^{\lambda}(i+1) .
$$

It follows from this, that the sum in (2.13.1) is a direct sum.

For $\left(i_{1}, \cdots, i_{g}\right) \in \Delta_{i, g}$, we consider a surjective $\overline{\mathscr{S}}^{\mathbf{p}}$-homomorphism

$\Psi: W^{\lambda^{[1]}}\left(i_{1}\right) \otimes \cdots \otimes W^{\lambda^{[g]}}\left(i_{g}\right) \rightarrow W^{\lambda^{[1]}}\left(i_{1}\right) / W^{\lambda^{[1]}}\left(i_{1}+1\right) \otimes \cdots \otimes W^{\lambda^{[g]}}\left(i_{g}\right) / W^{\lambda^{[g]}}\left(i_{g}+1\right)$

Then we have Ker $\Psi=\bar{Z}^{\lambda}(i+1) \cap\left(W^{\lambda^{[1]}}\left(i_{1}\right) \otimes \cdots \otimes W^{\lambda^{[g]}}\left(i_{g}\right)\right)$ under the setting in Corollary 2.12, By noting that (2.13.1) is a direct sum, we have

$$
\begin{aligned}
& \bar{Z}^{\lambda}(i) / \bar{Z}^{\lambda}(i+1) \\
& \quad \cong \bigoplus_{\left(i_{1}, \cdots, i_{g}\right) \in \Delta_{i, g}}\left(W^{\lambda^{[1]}}\left(i_{1}\right) / W^{\lambda^{[1]}}\left(i_{1}+1\right) \otimes \cdots \otimes W^{\lambda^{[g]}}\left(i_{g}\right) / W^{\lambda^{[g]}}\left(i_{g}+1\right)\right) .
\end{aligned}
$$

Since $\bar{L}^{\mu} \cong L^{\mu^{[1]}} \otimes \cdots \otimes L^{\mu^{[g]}}$, we have

$$
\begin{aligned}
& {\left[\bar{Z}^{\lambda}(i) / \bar{Z}^{\lambda}(i+1): \bar{L}^{\mu}\right]} \\
& \quad=\sum_{\left(i_{1}, \cdots, i_{g}\right) \in \Delta_{i, g}}\left[W^{\lambda^{[1]}}\left(i_{1}\right) / W^{\lambda^{[1]}}\left(i_{1}+1\right) \otimes \cdots \otimes W^{\lambda^{[g]}}\left(i_{g}\right) / W^{\lambda^{[g]}}\left(i_{g}+1\right): \bar{L}^{\mu}\right] \\
& \quad=\sum_{\left(i_{1}, \cdots, i_{g}\right) \in \Delta_{i, g}} \prod_{k=1}^{g}\left[W^{\lambda^{[k]}}\left(i_{k}\right) / W^{\lambda^{[k]}}\left(i_{k}+1\right): L^{\left.\mu^{[k]}\right]} .\right.
\end{aligned}
$$

The lemma is proved

We define $v$-decomposition numbers of $\mathscr{S}\left(\Lambda_{n_{k}}\right)$ for $k=1, \cdots, g$ by

$$
d_{\lambda^{[k]} \mu^{[k]}}(v):=\sum_{i_{k} \geq 0}\left[W^{\lambda^{[k]}}\left(i_{k}\right) / W^{\lambda^{[k]}}\left(i_{k}+1\right): L^{\mu^{[k]}}\right] \cdot v^{i}
$$

as in the case of $\mathscr{S}(\Lambda)$. Then we have the following theorem.

Theorem 2.14. For $\lambda, \mu \in \Lambda^{+}$such that $\alpha_{\mathbf{p}}(\lambda)=\alpha_{\mathbf{p}}(\mu)$, we have

$$
d_{\lambda \mu}(v)=\bar{d}_{\lambda \mu}(v)=\prod_{k=1}^{g} d_{\lambda^{[k]} \mu^{[k]}}(v) .
$$


Proof. The first equality follows from Theorem 2.8. So we prove the second equality. By Lemma 2.13, we have

$$
\begin{aligned}
\bar{d}_{\lambda \mu}(v) & =\sum_{i \geq 0}\left[\bar{Z}^{\lambda}(i) / \bar{Z}^{\lambda}(i+1): \bar{L}^{\mu}\right] \cdot v^{i} \\
& =\sum_{i \geq 0}\left(\sum_{\left(i_{1}, \cdots, i_{g}\right) \in \Delta_{i, g}} \prod_{k=1}^{g}\left[W^{\lambda^{[k]}}\left(i_{k}\right) / W^{\lambda^{[k]}}\left(i_{k}+1\right): L^{\left.\mu^{[k]}\right]}\right]\right) \cdot v^{i} \\
& =\sum_{i \geq 0} \sum_{\left(i_{1}, \cdots, i_{g}\right) \in \Delta_{i, g}}\left(\prod_{k=1}^{g}\left[W^{\lambda^{[k]}}\left(i_{k}\right) / W^{\lambda^{[k]}}\left(i_{k}+1\right): L^{\left.\mu^{[k]}\right]}\right] \cdot v^{i_{k}}\right) \\
& =\sum_{\left(i_{1}, \cdots, i_{g}\right) \in \mathbb{Z}_{\geq 0}^{g}}\left(\prod_{k=1}^{g}\left[W^{\lambda^{[k]}}\left(i_{k}\right) / W^{\lambda^{[k]}}\left(i_{k}+1\right): L^{\mu^{[k]}}\right] \cdot v^{i_{k}}\right) \\
& =\prod_{k=1}^{g} \sum_{i_{k} \geq 0}\left[W^{\lambda^{[k]}}\left(i_{k}\right) / W^{\lambda^{[k]}}\left(i_{k}+1\right): L^{\mu^{[k]}}\right] \cdot v^{i_{k}} \\
& =\prod_{k=1}^{g} d_{\lambda^{[k]} \mu^{[k]}}(v) .
\end{aligned}
$$

This proves the theorem.

\section{3. $v$-DeCOMPOSITION NUMBERS FOR ARIKI-KoIKE ALGEBRAS}

We keep the notation in the previous section. We consider the $v$-decomposition numbers of the Ariki-Koike algebra $\mathscr{H}$, and show that similar results hold as in the previous section.

3.1. Let $\omega=\left(-, \cdots,-,\left(1^{n}\right)\right)$ be the $r$-partition and let $T^{\omega}$ be the $\omega$-tableau of type $\omega$. Since $\varphi_{T^{\omega} T^{\omega}}$ is an identity map on $M^{\omega}$ and a zero map on $M^{\mu}$ for $\mu \in \Lambda$ such that $\mu \neq \omega, \varphi_{T^{\omega} T^{\omega}}$ is an idempotent in $\mathscr{S}$. Moreover, we see that $\varphi_{T^{\omega}{ }^{\omega}}{ }^{\omega} \mathscr{S}_{\varphi_{T}{ }^{\omega} T^{\omega}}=$ $\operatorname{Hom}_{\mathscr{H}}\left(M^{\omega}, M^{\omega}\right)=\operatorname{Hom}_{\mathscr{H}}(\mathscr{H}, \mathscr{H}) \cong \mathscr{H}$. It is well known that, for an $\mathscr{S}$-module $M, M \varphi_{T^{\omega} T^{\omega}}$ becomes a $\mathscr{H}$-module through the isomorphism $\varphi_{T^{\omega} T^{\omega}} \mathscr{S} \varphi_{T^{\omega} T^{\omega}} \cong$ $\mathscr{H}$. Then we can define a functor, the so-called "Schur functor", from the category of right $\mathscr{S}$-modules to the category of right $\mathscr{H}$-modules by $M \mapsto M \varphi_{T^{\omega} T^{\omega}} \omega$. The following facts are known by [JM, Proposition 2.17].

$$
\begin{array}{r}
W^{\lambda} \varphi_{T^{\omega} T^{\omega}} \cong S^{\lambda} \quad \text { as } \mathscr{H} \text {-modules } \quad\left(\lambda \in \Lambda^{+}\right), \\
L^{\mu} \varphi_{T^{\omega} T^{\omega}} \cong D^{\mu} \quad \text { as } \mathscr{H} \text {-modules }\left(\mu \in \Lambda^{+}\right), \\
{\left[W^{\lambda}: L^{\mu}\right]=\left[S^{\lambda}: D^{\mu}\right] \quad\left(\lambda, \mu \in \Lambda^{+} \text {such that } D^{\mu} \neq 0\right),}
\end{array}
$$

where $\left[S^{\lambda}: D^{\mu}\right]$ is the decomposition number of $D^{\mu}$ in $S^{\lambda}$.

3.2. One can define the Jantzen filtration of the Specht module $S^{\lambda}$ in a similar way as in the case of $W^{\lambda}$, and we use a similar notation for this case. Then one can define the $v$-decomposition number of $\mathscr{H}$, for $\lambda, \mu \in \Lambda^{+}$such that $D^{\mu} \neq 0$, by

$$
d_{\lambda \mu}^{\mathscr{H}}(v):=\sum_{i \geq 0}\left[S^{\lambda}(i) / S^{\lambda}(i+1): D^{\mu}\right] \cdot v^{i} .
$$

We have the following lemma. 
Lemma 3.3. Let $\lambda \in \Lambda^{+}$and $i \geq 0$. Under the isomorphism in (3.1.1), we have

$$
W^{\lambda}(i) \varphi_{T^{\omega} T^{\omega}}=S^{\lambda}(i) .
$$

Proof. It is clear that $W^{\lambda} \varphi_{T^{\omega} T^{\omega}}$ has a basis $\left\{\varphi_{T} \mid T \in \mathcal{T}_{0}(\lambda, \omega)\right\}$. We have a bijective correspondence between $\mathcal{T}_{0}(\lambda, \omega)$ and $\operatorname{Std}(\lambda)$ by $T \leftrightarrow \mathfrak{t}$ such that $\omega(\mathfrak{t})=T$. Moreover, under the isomorphism in (3.1.1), we have

$$
\begin{aligned}
& \varphi_{T} \varphi_{T^{\omega} T^{\omega}}= \begin{cases}m_{\mathfrak{t}} & \text { if } T \in \mathcal{T}_{0}(\lambda, \omega), \\
0 & \text { if } T \notin \mathcal{T}_{0}(\lambda, \omega),\end{cases} \\
& \left\langle\varphi_{S}, \varphi_{T}\right\rangle=\left\langle m_{\mathfrak{s}}, m_{\mathfrak{t}}\right\rangle_{\mathscr{H}} \quad \text { for } S=\omega(\mathfrak{s}), T=\omega(\mathfrak{t}) \in \mathcal{T}_{0}(\lambda, \omega),
\end{aligned}
$$

by a similar argument as in the proof of [M2, Theorem 4.18].

First, we show the inclusion $W^{\lambda}(i) \varphi_{T^{\omega} T^{\omega}} \subseteq S^{\lambda}(i)$. Take $x \in W_{R}^{\lambda}(i)$. Then $\left\langle x, \varphi_{T}\right\rangle \in \wp^{i}$ for any $T \in \mathcal{T}_{0}(\lambda)$. It follows that

$$
\left\langle x \cdot \varphi_{T^{\omega} T^{\omega}}, \varphi_{T}\right\rangle=\left\langle x, \varphi_{T} \cdot \varphi_{T^{\omega} T^{\omega}}\right\rangle \in \wp^{i} \quad \text { for any } T \in \mathcal{T}_{0}(\lambda) .
$$

This shows that

$$
\left\langle x \cdot \varphi_{T \omega} T^{\omega}, m_{\mathfrak{t}}\right\rangle_{\mathscr{H}} \in \wp^{i} \quad \text { for any } \mathfrak{t} \in \operatorname{Std}(\lambda)
$$

by (3.3.1) and (3.3.2). Hence $x \cdot \varphi_{T^{\omega}} T^{\omega} \in S_{R}^{\lambda}(i)$, and the claim follows by taking the quotient.

Next, we show the converse inclusion $W^{\lambda}(i) \varphi_{T^{\omega} T^{\omega}} \supseteq S^{\lambda}(i)$. Take $y \in S^{\lambda}(i)$. Then we have

$$
\left\langle y, m_{\mathfrak{s}}\right\rangle_{\mathscr{H}} \in \wp^{i} \quad \text { for any } \mathfrak{s} \in \operatorname{Std}(\lambda) .
$$

Write $y=\sum_{\mathfrak{t} \in \operatorname{Std}(\lambda)} r_{\mathfrak{t}} m_{\mathfrak{t}}$, and put $x=\sum_{T_{\in} \mathcal{T}_{0}(\lambda, \omega)} r_{\mathfrak{t}} \varphi_{T} \in W^{\lambda}$, where $T$ is the $\lambda$-tableau of type $\omega$ corresponding to $t$. Then we have $y=x \cdot \varphi_{T^{\omega}} T^{\omega}$, and

$$
\left\langle x, \varphi_{S}\right\rangle \in \wp^{i} \quad \text { for any } S \in \mathcal{T}_{0}(\lambda, \omega)
$$

by (3.3.1), (3.3.2) and (3.3.3). Since $\left\langle\varphi_{T}, \varphi_{S}\right\rangle=0$ if the type of $T$ is not the same as the type of $S$, we have

$$
\left\langle x, \varphi_{S}\right\rangle \in \wp^{i} \quad \text { for any } S \in \mathcal{T}_{0}(\lambda) .
$$

This shows that $x \in W_{R}^{\lambda}(i)$, and the claim follows. The lemma is proved.

This lemma implies the following proposition.

Proposition 3.4. Take $\lambda, \mu \in \Lambda^{+}$such that $D^{\mu} \neq 0$. Then for any $i \geq 0$, we have

$$
\left[W^{\lambda}(i) / W^{\lambda}(i+1): L^{\mu}\right]=\left[S^{\lambda}(i) / S^{\lambda}(i+1): D^{\mu}\right] .
$$

In particular, we have $d_{\lambda \mu}(v)=d_{\lambda \mu}^{\mathscr{H}}(v)$.

Proof. We consider the $\mathscr{S}$-module filtration

$$
W^{\lambda}(i)=W_{0} \supsetneqq W_{1} \supsetneqq \cdots \supsetneqq W_{k}=W^{\lambda}(i+1)
$$

such that $W_{j} / W_{j+1} \cong L^{\mu_{j}}$. By applying the Schur functor, together with Lemma 3.3. we have

$S^{\lambda}(i)=W^{\lambda}(i) \varphi_{T^{w} T^{w}} \supset W_{1} \varphi_{T^{\omega} T^{\omega}} \supset \cdots \supset W_{k} \varphi_{T^{\omega} T^{\omega}}=W^{\lambda}(i+1) \varphi_{T^{\omega} T^{\omega}}=S^{\lambda}(i+1)$, where $W_{j} \varphi_{T^{\omega} T^{\omega}} / W_{j+1} \varphi_{T^{\omega} T^{\omega}} \cong\left(W_{j} / W_{j+1}\right) \varphi_{T^{\omega} T^{\omega}} \cong L^{\mu_{j}} \varphi_{T^{\omega} T^{\omega}} \cong D^{\mu_{j}}$ by (3.1.2). The proposition follows from this. 
For $\lambda \in \Lambda^{+}$such that $\alpha_{\mathbf{p}}(\lambda)=\left(n_{1}, \cdots, n_{g}\right), \lambda^{[k]}$ is an $r_{k}$-partition of $n_{k}$. Then we have the Specht module $S^{\lambda^{[k]}}$ and its unique quotient $D^{\lambda^{[k]}}$ for the Ariki-Koike algebra $\mathscr{H}_{n_{k}, r_{k}}$. Moreover, for $\lambda, \mu \in \Lambda^{+}$such that $\alpha_{\mathbf{p}}(\lambda)=\alpha_{\mathbf{p}}(\mu)=\left(n_{1}, \cdots, n_{g}\right)$, we have the $v$-decomposition number $d_{\lambda^{[k]} \mu^{[k]}}^{\mathscr{H}}(v)$ for $\mathscr{H}_{n_{k} r_{k}}$. Combining Theorem 2.14 with Proposition 3.4, we have the following result.

Theorem 3.5. Let $\lambda, \mu \in \Lambda^{+}$such that $\alpha_{\mathbf{p}}(\lambda)=\alpha_{\mathbf{p}}(\mu)$. Assume that $D^{\mu} \neq 0$ and $D^{\mu^{[k]}} \neq 0$ for any $k=1, \cdots, g$. Then we have

$$
d_{\lambda \mu}^{\mathscr{H}}(v)=\prod_{k=1}^{g} d_{\lambda^{[k]} \mu^{[k]}}^{\mathscr{H}}(v) .
$$

\section{ACKNOWLEDGMENTS}

I would like to thank Toshiaki Shoji and Hyohe Miyachi for their helpful advice and many discussions.

\section{REFERENCES}

[A1] S. Ariki. On the decomposition numbers of the Hecke algebra of $G(m, 1, n)$. J. Math. Kyoto Univ. 36 (1996), 789-808. MR1443748 (98h:20012)

[A2] S. Ariki. On the classification of simple modules for cyclotomic Hecke algebras of type $G(m, 1, n)$ and Kleshchev multipartitions. Osaka J. Math. 38 (2001), 827-837. MR1864465 (2002i:20004)

[AM] S. Ariki and A. Mathas. The number of simple modules of the Hecke algebras of type $G(r, 1, n)$. Math. Z. 233 (2000), 601-623. MR.1750939 (2001e:20007)

[DJM] R. Dipper, G. James, and A. Mathas. Cyclotomic q-Schur algebras. Math. Z. 229 (1998), 385-416. MR1658581(2000a:20033)

[DR] J. Du and H. Rui. Based algebras and standard bases for quasi-hereditary algebras. Trans. Amer. Math. Soc. 350 (1998), 3207-3235. MR1603902 (99b:16027)

[GL] J. J. Graham and G. I. Lehrer. Cellular algebras. Invent. Math. 123 (1996), 1-34. MR:1376244 (97h:20016)

[Jac] N. Jacon. An algorithm for the computation of the decomposition matrices for Ariki-Koike algebras. J. Algebra 292 (2005), 100-109. MR.2166797 (2006g:20010)

[JM] G. James and A. Mathas. The Jantzen sum formula for cyclotomic $q$-Schur algebras. Trans. Amer. Math. Soc. 352 (2000), 5381-5404. MR.1665333 (2001b:16017)

[LLT] A. Lascoux, B. Leclerc, and J.-Y. Thibon. Hecke algebras at roots of unity and crystal bases of quantum affine algebras. Comm. Math. Phys. 181 (1996), 205-263. MR1410572 (97k:17019)

[LT] B. Leclerc and J.-Y. Thibon. Canonical bases of $q$-deformed Fock spaces. Internat. Math. Res. Notices, (1996) 447-456. MR.1399410 (97h:17023)

[M1] A. Mathas. Simple modules of Ariki-Koike algebras. In "Group representations: cohomology, group actions and topology", Proc. Sympos. Pure Math. vol. 63, Amer. Math. Soc., 1998, pp. 383-396. MR.1603195 (99d:20018)

[M2] A. Mathas. Iwahori-Hecke algebras and Schur algebras of the symmetric group, University Lecture Series Vol. 15, Amer. Math. Soc., 1999. MR1711316 (2001g:20006)

[M3] A. Mathas. The representation theory of the Ariki-Koike and cyclotomic $q$-Schur algebras. In "Representation theory of algebraic groups and quantum groups", Adv. Stud. Pure Math. Vol. 40, Math. Soc. Japan, Tokyo 2004, pp. 261-320. MR2074597 (2005f:20014)

[SW] T. Shoji and K. Wada. Cyclotomic $q$-Schur algebras associated to the Ariki-Koike algebra, Represent. Theory 14 (2010), 379-416.

[U] D. Uglov. Canonical bases of higher-level $q$-deformed Fock spaces and KazhdanLusztig polynomials. In "Physical combinatorics (Kyoto, 1999)", Progr. Math. vol. 191, Birkhäuser Boston, Boston, 2000, pp. 249-299 MR1768086 (2001k:17030) 
[VV] M. Varagnolo and E. Vasserot. On the decomposition matrices of the quantized Schur algebra. Duke Math. J. 100, (1999), 267-297. MR.1722955 (2001c:17029)

[Y] X. Yvonne. A conjecture for $q$-decomposition matrices of cyclotomic $v$-Schur algebras. J. Algebra, 304, (2006) 419-456. MR2256400 (2008d:16051)

Graduate School of Mathematics, Nagoya University, Chikusa-ku, Nagoya 464-8602, JAPAN

Current address: Research Institute for Mathematical Sciences, Kyoto University, Kyoto 6068502, Japan

E-mail address: wada@kurims.kyoto-u.ac.jp 